\title{
Homotopy Series Solutions to Time-Space Fractional Coupled Systems
}

\author{
Jin Zhang, ${ }^{1}$ Ming Cai, ${ }^{2}$ Bochao Chen, ${ }^{2}$ and Hui Wei ${ }^{2}$ \\ ${ }^{1}$ School of Mathematics, Jilin University, Changchun 130012, China \\ ${ }^{2}$ School of Mathematics and Statistics, Center for Mathematics and Interdisciplinary Sciences, Northeast Normal University, \\ Changchun, Jilin 130024, China
}

Correspondence should be addressed to Jin Zhang; jinzhang@jlu.edu.cn

Received 31 August 2017; Accepted 23 October 2017; Published 25 December 2017

Academic Editor: Jorge E. Macías-Díaz

Copyright (C) 2017 Jin Zhang et al. This is an open access article distributed under the Creative Commons Attribution License, which permits unrestricted use, distribution, and reproduction in any medium, provided the original work is properly cited.

\begin{abstract}
We apply the homotopy perturbation Sumudu transform method (HPSTM) to the time-space fractional coupled systems in the sense of Riemann-Liouville fractional integral and Caputo derivative. The HPSTM is a combination of Sumudu transform and homotopy perturbation method, which can be easily handled with nonlinear coupled system. We apply the method to the coupled Burgers system, the coupled KdV system, the generalized Hirota-Satsuma coupled KdV system, the coupled WBK system, and the coupled shallow water system. The simplicity and validity of the method can be shown by the applications and the numerical results.
\end{abstract}

\section{Introduction}

Fractional calculus, compared to integer calculus, was mentioned in a letter from L'Hospital to Leibniz in 1695. In the letter, L'Hospital raised a question, "what is the result of $\mathrm{d}^{n} y / \mathrm{d} x^{n}$ if $n=1 / 2$ ?" The answer of Leibniz was " $\mathrm{d}^{1 / 2} x$ will be equal to $x \sqrt{\mathrm{d} x: x}$. This is an apparent paradox, from which, one day useful consequences will be drawn" [1]. Furthermore, the generalization of this framework indicates that it is more appropriate to talk about integration and differentiation of arbitrary order, such as fractional order, real number order, and even complex number order just as the development of number system. Thus, there is a basic question: "what are the definitions of fractional integral and derivative?" Or "how to define the fractional integral and derivative?" More and more mathematicians focused on this problem, like Lagrange, Laplace, Fourier, and so on. Some different fractional integrals and derivatives have been given according to different needs, like Riemann-Liouville fractional integral, Caputo fractional derivative, Weyl fractional derivative, and so on [2]. But there are no uniform definitions of fractional integral and derivative, and the frequently used definitions are Riemann-Liouville integral and Caputo derivative.
Fractional differential equations arise in many engineering and scientific disciplines as the mathematical modeling of systems and processes in the fields of physics, chemistry, aerodynamics, electrodynamics of complex medium, polymer rheology, and so on, which involve derivatives of fractional order. Fractional differential equations also serve as an excellent tool for the description of hereditary properties of various materials and processes. In consequence, the subject of fractional differential equations is gaining much more attention. For example, in electromagnetism, Sebaa et al. [3] studied ultrasonic wave propagation in human cancellous bone by using fractional calculus to describe the viscous interactions between fluid and solid structure. In signal processing, Assaleh and Ahmad [4] proposed a new approach for speech signal modeling through using fractional calculus. Magin and Ovadia [5] molded the cardiac tissue electrode interface using fractional calculus. In control theory, Suarez et al. [6] applied fractional controllers to the path-tracking problem in an autonomous electric vehicle. In fluid mechanics, Kulish and Lage [7] applied fractional calculus to the solution of time-dependent, viscous-diffusion fluid mechanics problems. 
In this paper, we intend to construct the approximate solutions to the nonlinear time-space fractional coupled systems. There are many effective methods to solve this problem, like Adomian decomposition method [8-10], variation iteration method [11], differential transform method [12], residual power series method [13, 14], iteration method [15], homotopy perturbation method [16], homotopy analysis method [17], and so on. Furthermore, for the nonlinear problem, the multiple exp-function method [18, 19], the transformed rational function method [20-22], and invariant subspace method $[23,24]$ are three systematical approaches to handle the nonlinear terms. The first one is to propose the exact solution of nonlinear partial differential equations by using rational function transformations. Its key point is to search for rational solutions to variable-coefficient ordinary differential equations transformed from given partial differential equations. The second one is to consider the form of solution as rational exponential functions with unknown coefficients whose advantage is direct applicability to underlying equation. The invariant subspace method is refined to present more unity and more diversity of exact solutions to evolution equations. The key idea is to take subspaces of solutions to linear ordinary differential equations as invariant subspaces that evolution equations admit. Motivated by these fruitful results, Singh et al. [25] proposed the homotopy perturbation Sumudu transform method based on the homotopy perturbation method and Sumudu transform method and applied it to nonlinear partial differential equations. The HPSTM was extended to the time-fractional PDEs in $[26,27]$. It is worth mentioning that the HPSTM is applied without any using of Adomian polynomials, over restrictive assumption or linearization, and is capable of reducing the volume of computational work as compared to the classical numerical methods while still maintaining the high accuracy of the result. Meanwhile, it is appropriate not only for strongly nonlinear system but also for weakly nonlinear system.

The rest of the paper is organized as follows. In Section 2, we introduce some concepts on fractional calculus and the Sumudu transform. In Section 3, we illustrate the basic idea of HPSTM which is applied to the time-space fractional coupled systems. In Section 4, we apply HPSTM to obtain fractional power series solutions of nonlinear time-space fractional coupled systems with initial values, and some numerical results are presented as well.

\section{Preliminaries}

Definition 1 (see [2]). A real function $f(x), x>0$ is said to be in the space $C_{\mu}, \mu \in \mathbb{R}$, if there exists a real number $\rho>\mu$, such that $f(x)=x^{\rho} f_{1}(x)$, where $f_{1}(x) \in C[0, \infty)$, and it is said that $f(x) \in C_{\mu}^{n}$, if $f^{(n)}(x) \in C_{\mu}, n \in \mathbb{N}$.

Definition 2 (see [2]). The fractional integral of $f(t)$ in the Riemann-Liouville (left-sided) sense is defined as

$$
\begin{aligned}
& I_{t}^{\alpha} f(t) \\
& \quad:= \begin{cases}\frac{1}{\Gamma(\alpha)} \int_{0}^{t}(t-\tau)^{\alpha-1} f(\tau) \mathrm{d} \tau, & \alpha>0, t>\tau \geqslant 0 ; \\
f(t), & \alpha=0,\end{cases}
\end{aligned}
$$

where $\alpha \geqslant 0, f \in C_{\mu}, \mu \geqslant-1$, and $\Gamma$ is the Gamma function.

Definition 3 (see [2]). The fractional integral of $f(x)$ in the Riemann-Liouville sense is defined as

$$
\begin{aligned}
& I_{x}^{\alpha} f(x) \\
& := \begin{cases}\frac{1}{\Gamma(\alpha)} \int_{-\infty}^{x}(x-\tau)^{\alpha-1} f(\tau) \mathrm{d} \tau, & \alpha>0,-\infty<x<\infty ; \\
f(x), & \alpha=0,\end{cases}
\end{aligned}
$$

where $\alpha \geqslant 0, f \in C_{\mu}, \mu \geqslant-1$, and $\Gamma$ is the Gamma function.

Definition 4 (see [2]). The Caputo (left-sided) fractional derivative operator of order $\alpha \geq 0$, of a function $f \in C_{\mu}^{n}(\mu \geqslant$ $-1, n \in \mathbb{N}$ ), is defined as

$$
\begin{aligned}
& D_{t}^{\alpha} f(t) \\
& := \begin{cases}\frac{1}{\Gamma(n-\alpha)} \int_{0}^{t}(t-\tau)^{n-\alpha-1} f^{(n)}(\tau) \mathrm{d} \tau, & n-1<\alpha<n, t>0 ; \\
\frac{\mathrm{d}^{n} f(t)}{\mathrm{d} t^{n}}, & \alpha=n .\end{cases}
\end{aligned}
$$

Definition 5 (see [2]). The Caputo fractional derivative operator of order $\alpha \geq 0$, of a function $f \in C_{\mu}^{n}(\mu \geqslant-1, n \in \mathbb{N})$, is defined as

$$
D_{x}^{\alpha} f(x):= \begin{cases}\frac{1}{\Gamma(n-\alpha)} \int_{-\infty}^{x}(x-\tau)^{n-\alpha-1} f^{(n)}(\tau) \mathrm{d} \tau, & n-1<\alpha<n,-\infty<x<\infty \\ \frac{\mathrm{d}^{n} f(x)}{\mathrm{d} x^{n}}, & \alpha=n .\end{cases}
$$

Lemma 6 (see [28]). If $m-1<\alpha \leq m, m \in \mathbb{N}$, and $f \in C_{\mu}^{m}$, $\mu \geq-1$, one has

$$
D_{t}^{\alpha} I_{t}^{\alpha} f(t)=f(t)
$$

$$
I_{t}^{\alpha} D_{t}^{\alpha} f(t)=f(t)-\sum_{k=0}^{m-1} f^{(k)}(0) \frac{t^{k}}{k !}
$$


In 1998, a new integral transform, named Sumudu transform, was introduced by Watugala [29] to study solutions of ordinary differential equations in control engineering problems. The Sumudu transform is defined over the set of functions $A=\left\{f(t): \exists M, \tau_{1}, \tau_{2}>0\right.$, s.t. $|f(t)|<$ $M e^{|t| / \tau_{j}}$, if $\left.t \in(-1)^{j} \times[0,+\infty)\right\}$ by the following formula:

$$
F(u)=S[f(t)]=\int_{0}^{\infty} f(u t) e^{-t} \mathrm{~d} t, \quad u \in\left(-\tau_{1}, \tau_{2}\right) .
$$

Property 7 (see [30]). (i) The Sumudu transform satisfies linear property; that is,

$$
S[a f(t)+b h(t)]=a S[f(t)]+b S[h(t)],
$$

where $a, b$ are constants.

(ii)

$$
S\left[t^{n}\right]=u^{n} \Gamma(n+1), \quad n \in \mathbb{N} .
$$

Lemma 8 (see [31]). The Sumudu transform of the Caputo fractional derivative is

$$
\begin{aligned}
S\left[D_{t}^{\alpha} f\right](t)=u^{-\alpha} S[f(t)]-\sum_{k=0}^{m} u^{-\alpha+k} f^{(k)}(0), & \\
& m<\alpha \leq m+1, m \in \mathbb{N} .
\end{aligned}
$$

\section{Homotopy Perturbation Sumudu Transform Method}

In this section, to illustrate the basic idea of this method, we consider a general nonhomogeneous fractional partial differential coupled system

$$
\begin{aligned}
& D_{t}^{\alpha} U^{1}(x, t)+\mathscr{R}^{1}\left(U^{1}, U^{2}, U^{3}\right)+\mathscr{N}^{1}\left(U^{1}, U^{2}, U^{3}\right) \\
& \quad=g^{1}(x, t), \\
& D_{t}^{\beta} U^{2}(x, t)+\mathscr{R}^{2}\left(U^{1}, U^{2}, U^{3}\right)+\mathscr{N}^{2}\left(U^{1}, U^{2}, U^{3}\right) \\
& \quad=g^{2}(x, t), \\
& D_{t}^{\gamma} U^{3}(x, t)+\mathscr{R}^{3}\left(U^{1}, U^{2}, U^{3}\right)+\mathscr{N}^{3}\left(U^{1}, U^{2}, U^{3}\right) \\
& \quad=g^{3}(x, t),
\end{aligned}
$$

with the initial conditions

$$
\begin{aligned}
& U^{1}(x, 0)=f^{1}(x), \\
& U^{2}(x, 0)=f^{2}(x), \\
& U^{3}(x, 0)=f^{3}(x),
\end{aligned}
$$

where $\alpha, \beta, \gamma \in(0,1], \mathscr{R}^{i}, \mathscr{N}^{i}, i=1,2,3$, denote linear differential operators and nonlinear differential operators, respectively, and $g^{i}(x, t)$ are the source terms. Applying the Sumudu transform on both sides of (10) yields

$$
\begin{gathered}
S\left[D_{t}^{\alpha} U^{1}(x, t)\right]+S\left[\mathscr{R}^{1}\left(U^{1}, U^{2}, U^{3}\right)\right] \\
+S\left[\mathscr{N}^{1}\left(U^{1}, U^{2}, U^{3}\right)\right]=S\left[g^{1}(x, t)\right], \\
S\left[D_{t}^{\beta} U^{2}(x, t)\right]+S\left[\mathscr{R}^{2}\left(U^{1}, U^{2}, U^{3}\right)\right] \\
+S\left[\mathcal{N}^{2}\left(U^{1}, U^{2}, U^{3}\right)\right]=S\left[g^{2}(x, t)\right], \\
S\left[D_{t}^{\gamma} U^{3}(x, t)\right]+S\left[\mathscr{R}^{3}\left(U^{1}, U^{2}, U^{3}\right)\right] \\
+S\left[\mathscr{N}^{3}\left(U^{1}, U^{2}, U^{3}\right)\right]=S\left[g^{3}(x, t)\right] .
\end{gathered}
$$

It follows from the property of the Sumudu transform in (9) that

$$
\begin{gathered}
S\left[U^{1}(x, t)\right]=f^{1}(x)-u^{\alpha}\left(S\left[\mathscr{R}^{1}\left(U^{1}, U^{2}, U^{3}\right)\right]\right. \\
\left.+S\left[\mathscr{N}^{1}\left(U^{1}, U^{2}, U^{3}\right)\right]\right)+u^{\alpha} S\left[g^{1}(x, t)\right], \\
S\left[U^{2}(x, t)\right]=f^{2}(x)-u^{\beta}\left(S\left[\mathscr{R}^{2}\left(U^{1}, U^{2}, U^{3}\right)\right]\right. \\
\left.\quad+S\left[\mathscr{N}^{2}\left(U^{1}, U^{2}, U^{3}\right)\right]\right)+u^{\beta} S\left[g^{2}(x, t)\right], \\
S\left[U^{3}(x, t)\right]=f^{3}(x)-u^{\gamma}\left(S\left[\mathscr{R}^{3}\left(U^{1}, U^{2}, U^{3}\right)\right]\right. \\
\left.\quad+S\left[\mathscr{N}^{3}\left(U^{1}, U^{2}, U^{3}\right)\right]\right)+u^{\gamma} S\left[g^{3}(x, t)\right] .
\end{gathered}
$$

Furthermore, applying the inverse Sumudu transform $S^{-1}$ on both sides of (13) yields

$$
\begin{aligned}
& U^{1}(x, t)=M^{1}(x, t)-S^{-1}\left[u ^ { \alpha } S \left[\mathscr{R}^{1}\left(U^{1}, U^{2}, U^{3}\right)\right.\right. \\
& \left.\left.+\mathscr{N}^{1}\left(U^{1}, U^{2}, U^{3}\right)\right]\right], \\
& U^{2}(x, t)=M^{2}(x, t)-S^{-1}\left[u ^ { \beta } S \left[\mathscr{R}^{2}\left(U^{1}, U^{2}, U^{3}\right)\right.\right. \\
& \left.\left.\quad+\mathscr{N}^{2}\left(U^{1}, U^{2}, U^{3}\right)\right]\right], \\
& U^{3}(x, t)=M^{3}(x, t)-S^{-1}\left[\mathcal { u } ^ { \gamma } S \left[\mathscr{R}^{3}\left(U^{1}, U^{2}, U^{3}\right)\right.\right. \\
& \left.\left.+\mathscr{N}^{3}\left(U^{1}, U^{2}, U^{3}\right)\right]\right],
\end{aligned}
$$

where $M^{i}(x, t), i=1,2,3$, represent the terms arising from the source terms and prescribed initial conditions; that is,

$$
\begin{aligned}
& M^{1}(x, t)=S^{-1}\left[f^{1}(x)+u^{\alpha} S\left[g^{1}(x, t)\right]\right], \\
& M^{2}(x, t)=S^{-1}\left[f^{2}(x)+u^{\beta} S\left[g^{2}(x, t)\right]\right], \\
& M^{3}(x, t)=S^{-1}\left[f^{3}(x)+u^{\gamma} S\left[g^{3}(x, t)\right]\right] .
\end{aligned}
$$

Let us construct the homotopy perturbation equations

$$
\begin{aligned}
& U^{1}(x, t)=M^{1}(x, t)-p \times S^{-1}\left[u ^ { \alpha } S \left[\mathscr{R}^{1}\left(U^{1}, U^{2}, U^{3}\right)\right.\right. \\
& \left.\left.\quad+\mathscr{N}^{1}\left(U^{1}, U^{2}, U^{3}\right)\right]\right]
\end{aligned}
$$




$$
\begin{aligned}
& U^{2}(x, t)=M^{2}(x, t)-p \times S^{-1}\left[u ^ { \beta } S \left[\mathscr{R}^{2}\left(U^{1}, U^{2}, U^{3}\right)\right.\right. \\
& \left.\left.\quad+\mathscr{N}^{2}\left(U^{1}, U^{2}, U^{3}\right)\right]\right], \\
& U^{3}(x, t)=M^{3}(x, t)-p \times S^{-1}\left[u ^ { \gamma } S \left[\mathscr{R}^{3}\left(U^{1}, U^{2}, U^{3}\right)\right.\right. \\
& \left.\left.+\mathscr{N}^{3}\left(U^{1}, U^{2}, U^{3}\right)\right]\right],
\end{aligned}
$$

where homotopy parameter $p \in[0,1]$. Suppose that $U^{i}(x, t)$ and the nonlinear terms $\mathscr{N}^{j} U^{i}(x, t)$ can be written as

$$
\begin{aligned}
U^{i}(x, t) & =\sum_{n=0}^{\infty} p^{n} U_{n}^{i}(x, t), \quad i=1,2,3, \\
\mathcal{N}^{j}\left(U^{1}, U^{2}, U^{3}\right) & =\sum_{n=0}^{\infty} p^{n} H_{n}^{j}\left(U^{1}, U^{2}, U^{3}\right),
\end{aligned}
$$

$$
j=1,2,3,
$$

where the coefficient polynomials $U_{n}^{i}$ can be determined below and $H_{n}^{j}\left(U^{1}, U^{2}, U^{3}\right)$ are given by the following formulae:

$$
\begin{aligned}
& H_{n}^{j}\left(U^{1}, U^{2}, U^{3}\right)=\frac{1}{n !} \\
& \left.\cdot \frac{\partial^{n}}{\partial p^{n}}\left(\mathscr{N}^{j}\left(\sum_{k=0}^{\infty} p^{k} U_{k}^{1}, \sum_{k=0}^{\infty} p^{k} U_{k}^{2}, \sum_{k=0}^{\infty} p^{k} U_{k}^{3}\right)\right)\right|_{p=0} \\
& =\frac{1}{n !} \frac{\partial^{n}}{\partial p^{n}}\left(\mathcal{N}^{j}\left(\sum_{k=0}^{n} p^{k} U_{k}^{1}, \sum_{k=0}^{n} p^{k} U_{k}^{2}, \sum_{k=0}^{n} p^{k} U_{k}^{3}\right)\right. \\
& \left.+\mathcal{N}^{j}\left(\sum_{k=n+1}^{\infty} p^{k} U_{k}^{1}, \sum_{k=n+1}^{\infty} p^{k} U_{k}^{2}, \sum_{k=n+1}^{\infty} p^{k} U_{k}^{3}\right)\right)\left.\right|_{p=0} \\
& \quad=\frac{1}{n !}
\end{aligned}
$$

$$
\begin{array}{r}
\left.\frac{\partial^{n}}{\partial p^{n}}\left(\mathscr{N}^{j}\left(\sum_{k=0}^{n} p^{k} U_{k}^{1}, \sum_{k=0}^{n} p^{k} U_{k}^{2}, \sum_{k=0}^{n} p^{k} U_{k}^{3}\right)\right)\right|_{p=0}, \\
j=1,2,3 .
\end{array}
$$

Substituting (17) into (16) gives

$$
\begin{aligned}
& \sum_{n=0}^{\infty} p^{n} U_{n}^{1}(x, t)=M^{1}(x, t)-p \\
& \times S^{-1}\left[u ^ { \alpha } S \left[\mathscr{R}^{1}\left(\sum_{k=0}^{\infty} p^{k} U_{k}^{1}, \sum_{k=0}^{\infty} p^{k} U_{k}^{2}, \sum_{k=0}^{\infty} p^{k} U_{k}^{3}\right)\right.\right. \\
& \left.\left.+\sum_{n=0}^{\infty} p^{n} H_{n}^{1}\left(\sum_{k=0}^{n} p^{k} U_{k}^{1}, \sum_{k=0}^{n} p^{k} U_{k}^{2}, \sum_{k=0}^{n} p^{k} U_{k}^{3}\right)\right]\right], \\
& \sum_{n=0}^{\infty} p^{n} U_{n}^{2}(x, t)=M^{2}(x, t)-p \\
& \quad \times S^{-1}\left[u ^ { \beta } S \left[\mathscr{R}^{2}\left(\sum_{k=0}^{\infty} p^{k} U_{k}^{1}, \sum_{k=0}^{\infty} p^{k} U_{k}^{2}, \sum_{k=0}^{\infty} p^{k} U_{k}^{3}\right)\right.\right. \\
& \left.\left.+\sum_{n=0}^{\infty} p^{n} H_{n}^{2}\left(\sum_{k=0}^{n} p^{k} U_{k}^{1}, \sum_{k=0}^{n} p^{k} U_{k}^{2}, \sum_{k=0}^{n} p^{k} U_{k}^{3}\right)\right]\right], \\
& \sum_{n=0}^{\infty} p^{n} U_{n}^{3}(x, t)=M^{3}(x, t)-p \\
& \quad \times S^{-1}\left[u ^ { \gamma } S \left[\mathscr{R}^{3}\left(\sum_{k=0}^{\infty} p^{k} U_{k}^{1}, \sum_{k=0}^{\infty} p^{k} U_{k}^{2}, \sum_{k=0}^{\infty} p^{k} U_{k}^{3}\right)\right.\right. \\
& \left.\left.\quad+\sum_{n=0}^{\infty} p^{n} H_{n}^{3}\left(\sum_{k=0}^{n} p^{k} U_{k}^{1}, \sum_{k=0}^{n} p^{k} U_{k}^{2}, \sum_{k=0}^{n} p^{k} U_{k}^{3}\right)\right]\right] .
\end{aligned}
$$

Comparing the coefficients of $p$, we obtain the following recurrence equations:

$$
\begin{aligned}
& p^{0}: \quad U_{0}^{1}(x, t)=M^{1}(x, t), \\
& U_{0}^{2}(x, t)=M^{2}(x, t), \\
& U_{0}^{3}(x, t)=M^{3}(x, t) \text {, } \\
& p^{n}: \quad U_{n}^{1}(x, t)=-S^{-1}\left[u^{\alpha} S\left[\mathscr{R}^{1}\left(\sum_{r=0}^{n-1} \sum_{s=0}^{n-1-r} U_{r}^{1} U_{s}^{2} U_{n-1-r-s}^{3}\right)+H_{n-1}^{1}\left(\sum_{k=0}^{n} p^{k} U_{k}^{1}, \sum_{k=0}^{n} p^{k} U_{k}^{2}, \sum_{k=0}^{n} p^{k} U_{k}^{3}\right)\right]\right], \\
& U_{n}^{2}(x, t)=-S^{-1}\left[u^{\beta} S\left[\mathscr{R}^{2}\left(\sum_{r=0}^{n-1} \sum_{s=0}^{n-1-r} U_{r}^{1} U_{s}^{2} U_{n-1-r-s}^{3}\right)+H_{n-1}^{2}\left(\sum_{k=0}^{n} p^{k} U_{k}^{1}, \sum_{k=0}^{n} p^{k} U_{k}^{2}, \sum_{k=0}^{n} p^{k} U_{k}^{3}\right)\right]\right], \\
& U_{n}^{3}(x, t)=-S^{-1}\left[u^{\gamma} S\left[\mathscr{R}^{3}\left(\sum_{r=0}^{n-1} \sum_{s=0}^{n-1-r} U_{r}^{1} U_{s}^{2} U_{n-1-r-s}^{3}\right)+H_{n-1}^{3}\left(\sum_{k=0}^{n} p^{k} U_{k}^{1}, \sum_{k=0}^{n} p^{k} U_{k}^{2}, \sum_{k=0}^{n} p^{k} U_{k}^{3}\right)\right]\right] \text {, }
\end{aligned}
$$


According to the homotopy perturbation method, we assume that the solution of (10)-(11) can be written as

$$
\begin{array}{r}
V^{i}(x, t)=\lim _{n \rightarrow \infty}\left(U_{0}^{i}+U_{1}^{i} p+U_{2}^{i} p^{2}+\cdots+U_{n}^{i} p^{n}\right), \\
i=1,2,3 .
\end{array}
$$

Setting $p \rightarrow 1$, the approximate solution to (10)-(11) is

$$
\begin{aligned}
U^{i}(x, t) & =\lim _{p \rightarrow 1} V^{i}\left(x_{1}, \ldots, x_{m}, t\right) \\
& =\lim _{n \rightarrow \infty}\left(U_{0}^{i}+U_{1}^{i}+U_{2}^{i}+\cdots+U_{n}^{i}\right), \\
& i=1,2,3 .
\end{aligned}
$$

The convergence of series (21) depends on the nonlinear differential operator $\mathcal{N}$. Generally, the derivative with respect to $U$ of the nonlinear part in the splitting must be sufficiently small, since the parameter $p$ may be relatively large; in fact we take $p \rightarrow 1$. The series is convergent for most cases [32].

Remark 9. HPSTM is applied to construct homotophy series solutions for fractional coupled systems, which has not too many overstrict assumptions compared to some classical methods.

\section{Application of HPSTM to Time-Space Fractional Coupled Systems}

In this section, we apply HPSTM to nonlinear time-space fractional coupled systems with initial conditions.

4.1. The Time-Space Fractional Coupled Burgers System. The Burgers equation is one of the most important partial differential equations from fluid mechanics, which not only describes many phenomena, for example, modeling the motion of turbulence [33], but also has many applications in science and engineering [34]. Here we apply HPSTM to solve the following nonlinear time-space fractional coupled Burgers system:

$$
\begin{aligned}
& D_{t}^{\alpha} U-D_{x}^{2} U-2 U D_{x}^{\beta} U+D_{x}(U V)=0, \\
& D_{t}^{\gamma} V-D_{x}^{2} V-2 V D_{x}^{\delta} V+D_{x}(U V)=0,
\end{aligned}
$$

with the initial conditions

$$
\begin{aligned}
& U(x, 0)=\sin x, \\
& V(x, 0)=\sin x,
\end{aligned}
$$

where $0<\alpha, \beta, \delta, \gamma \leq 1,(x, t) \in \mathbb{R} \times[0, \infty)$.

Applying the Sumudu transform on both sides of (23) with the initial conditions, we can obtain

$$
\begin{aligned}
& S[U(x, t)]=\sin x+u^{\alpha}\left(S \left[D_{x}^{2} U(x, t)\right.\right. \\
& \left.\left.\quad+2 U(x, t) D_{x}^{\beta} U(x, t)-D_{x}(U(x, t) V(x, t))\right]\right), \\
& S[V(x, t)]=\sin x+v^{\gamma}\left(S \left[D_{x}^{2} V(x, t)\right.\right. \\
& \left.\left.\quad+2 V(x, t) D_{x}^{\delta} V(x, t)-D_{x}(U(x, t) V(x, t))\right]\right) .
\end{aligned}
$$

The inverse Sumudu transform of (25) implies that

$$
\begin{aligned}
& U(x, t)=\sin x+S^{-1}\left[u ^ { \alpha } \left(S \left[D_{x}^{2} U(x, t)\right.\right.\right. \\
& \left.\left.\left.\quad+2 U(x, t) D_{x}^{\beta} U(x, t)-D_{x}(U(x, t) V(x, t))\right]\right)\right], \\
& V(x, t)=\sin x+S^{-1}\left[v ^ { \gamma } \left(S \left[D_{x}^{2} V(x, t)\right.\right.\right. \\
& \left.\left.\left.\quad+2 V(x, t) D_{x}^{\delta} V(x, t)-D_{x}(U(x, t) V(x, t))\right]\right)\right] .
\end{aligned}
$$

Now applying the homotopy perturbation method gives

$$
\begin{aligned}
& \sum_{n=0}^{\infty} p^{n} U_{n}(x, t)=\sin x+p \\
& \times S^{-1}\left[u ^ { \alpha } S \left[D_{x}^{2}\left(\sum_{n=0}^{\infty} p^{n} U_{n}(x, t)\right)\right.\right. \\
& \left.\left.+\sum_{n=0}^{\infty} p^{n} H_{n}^{U}(x, t)\right]\right], \\
& \sum_{n=0}^{\infty} p^{n} V_{n}(x, t)=\sin x+p \\
& \quad \times S^{-1}\left[v ^ { \gamma } S \left[D_{x}^{2}\left(\sum_{n=0}^{\infty} p^{n} V_{n}(x, t)\right)\right.\right. \\
& \left.\left.+\sum_{n=0}^{\infty} p^{n} H_{n}^{V}(x, t)\right]\right],
\end{aligned}
$$

where $H_{n}^{U}(x, t)$ and $H_{n}^{V}(x, t)$ are polynomials which denote the homotopy coefficients of the nonlinear term and are given by

$$
\begin{aligned}
\sum_{n=0}^{\infty} p^{n} H_{n}^{U}(x, t)= & 2 U(x, t) D_{x}^{\beta} U(x, t) \\
& -D_{x}(U(x, t) V(x, t)), \\
\sum_{n=0}^{\infty} p^{n} H_{n}^{V}(x, t)= & 2 V(x, t) D_{x}^{\delta} V(x, t) \\
& -D_{x}(U(x, t) V(x, t)) .
\end{aligned}
$$

Set

$$
\begin{aligned}
& U_{n}(x, t):=\widetilde{U}_{n}(x) \frac{t^{n \alpha}}{\Gamma(n \alpha+1)}, \\
& V_{n}(x, t):=\widetilde{V}_{n}(x) \frac{t^{n \gamma}}{\Gamma(n \gamma+1)},
\end{aligned}
$$

and then

$$
\begin{gathered}
H_{n}^{U}(x, t)=2 \sum_{i=0}^{n} \widetilde{U}_{i}(x) D_{x}^{\beta} \widetilde{U}_{n-i}(x) \\
\cdot \frac{t^{n \alpha}}{\Gamma(i \alpha+1) \Gamma((n-i) \alpha+1)} \\
-\sum_{i=0}^{n} D_{x}\left(\widetilde{U}_{i}(x) \widetilde{V}_{n-i}(x)\right)
\end{gathered}
$$




$$
\begin{gathered}
\cdot \frac{t^{n \alpha}}{\Gamma(i \alpha+1) \Gamma((n-i) \alpha+1)}, \\
H_{n}^{V}(x, t)=2 \sum_{i=0}^{n} \widetilde{V}_{i}(x) D_{x}^{\delta} \widetilde{V}_{n-i}(x) \\
\cdot \frac{t^{n \gamma}}{\Gamma(i \gamma+1) \Gamma((n-i) \gamma+1)}
\end{gathered}
$$

$$
\begin{aligned}
& -\sum_{i=0}^{n} D_{x}\left(\widetilde{U}_{i}(x) \widetilde{V}_{n-i}(x)\right) \\
& \cdot \frac{t^{n \gamma}}{\Gamma(i \gamma+1) \Gamma((n-i) \gamma+1)} .
\end{aligned}
$$

Comparing the coefficients of $p$, this gives

$$
\begin{aligned}
p^{0}: & U_{0}(x, t)=\widetilde{U}_{0}(x)=\sin x, \\
& V_{0}(x, t)=\widetilde{V}_{0}(x)=\sin x, \\
p^{1}: \quad & U_{1}(x, t)=S^{-1}\left[u^{\alpha} S\left[D_{x}^{2} U_{0}(x, t)+H_{0}^{u}(x, t)\right]\right]=S^{-1}\left[u^{\alpha} S\left[D_{x}^{2} \widetilde{U}_{0}(x)+2 \widetilde{U}_{0}(x) D_{x}^{\beta} \widetilde{U}_{0}(x)-D_{x}\left(\widetilde{U}_{0}(x) \widetilde{V}_{0}(x)\right)\right]\right] \\
& =\frac{t^{\alpha}}{\Gamma(\alpha+1)}\left\{D_{x}^{2} \widetilde{U}_{0}(x)+2 \widetilde{U}_{0}(x) D_{x}^{\beta} \widetilde{U}_{0}(x)-D_{x}\left(\widetilde{U}_{0}(x) \widetilde{V}_{0}(x)\right)\right\}=\frac{t^{\alpha}}{\Gamma(\alpha+1)} \widetilde{U}_{1}(x), \\
& V_{1}(x, t)=S^{-1}\left[u^{\gamma} S\left[D_{x}^{2} V_{0}(x, t)+H_{0}^{v}(x, t)\right]\right]=S^{-1}\left[u^{\gamma} S\left[D_{x}^{2} \widetilde{V}_{0}(x)+2 \widetilde{V}_{0}(x) D_{x}^{\delta} \widetilde{V}_{0}(x)-D_{x}\left(\widetilde{U}_{0}(x) \widetilde{V}_{0}(x)\right)\right]\right] \\
& =\frac{t^{\gamma}}{\Gamma(\gamma+1)}\left\{D_{x}^{2} \widetilde{V}_{0}(x)+2 \widetilde{V}_{0}(x) D_{x}^{\delta} \widetilde{U}_{0}(x)-D_{x}\left(\widetilde{U}_{0}(x) \widetilde{V}_{0}(x)\right)\right\}=\frac{t^{\gamma}}{\Gamma(\gamma+1)} \widetilde{V}_{1}(x) .
\end{aligned}
$$

Generally, we have

$$
\begin{aligned}
p^{k}: & U_{k}(x, t) \\
& =\frac{t^{k \alpha}}{\Gamma(k \alpha+1)}\left\{D_{x}^{2} \widetilde{U}_{k-1}(x)+\sum_{i=0}^{k-1} \frac{\Gamma((k-1) \alpha+1)}{\Gamma(i \alpha+1) \Gamma((k-i-1) \alpha+1)}\left(2 \widetilde{U}_{i}(x) D_{x}^{\beta} \widetilde{U}_{k-1-i}(x)-D_{x}\left(\widetilde{U}_{i}(x) \widetilde{V}_{k-1-i}(x)\right)\right)\right\} \\
& =\frac{t^{k \alpha}}{\Gamma(k \alpha+1)} \widetilde{U}_{k}(x), \\
& V_{k}(x, t) \\
& =\frac{t^{k \gamma}}{\Gamma(k \gamma+1)}\left\{D_{x}^{2} \widetilde{V}_{k-1}(x)+\sum_{i=0}^{k-1} \frac{\Gamma((k-1) \gamma+1)}{\Gamma(i \gamma+1) \Gamma((k-i-1) \gamma+1)}\left(2 \widetilde{V}_{i}(x) D_{x}^{\delta} \widetilde{V}_{k-1-i}(x)-D_{x}\left(\widetilde{U}_{i}(x) \widetilde{V}_{k-1-i}(x)\right)\right)\right\} \\
& =\frac{t^{k \gamma}}{\Gamma(k \gamma+1)} \widetilde{V}_{k}(x),
\end{aligned}
$$

where

$$
\begin{aligned}
& \widetilde{U}_{k}(x)=D_{x}^{2} \widetilde{U}_{k-1}(x) \\
& +\sum_{i=0}^{k-1} \frac{\Gamma((k-1) \alpha+1)}{\Gamma(i \alpha+1) \Gamma((k-i-1) \alpha+1)}\left(2 \widetilde{U}_{i}(x)\right. \\
& \left.\cdot D_{x}^{\beta} \widetilde{U}_{k-1-i}(x)-D_{x}\left(\widetilde{U}_{i}(x) \widetilde{V}_{k-1-i}(x)\right)\right), \\
& \widetilde{V}_{k}(x)=D_{x}^{2} \widetilde{V}_{k-1}(x) \\
& +\sum_{i=0}^{k-1} \frac{\Gamma((k-1) \gamma+1)}{\Gamma(i \gamma+1) \Gamma((k-i-1) \gamma+1)}\left(2 \widetilde{V}_{i}(x)\right. \\
& \left.\cdot D_{x}^{\delta} \widetilde{V}_{k-1-i}(x)-D_{x}\left(\widetilde{U}_{i}(x) \widetilde{V}_{k-1-i}(x)\right)\right) .
\end{aligned}
$$

Particularly, when $\alpha=\gamma=\beta=\delta=1$, the exact solution of (23) is

$$
\begin{aligned}
& U(x, t)=e^{-t} \sin x, \\
& V(x, t)=e^{-t} \sin x .
\end{aligned}
$$




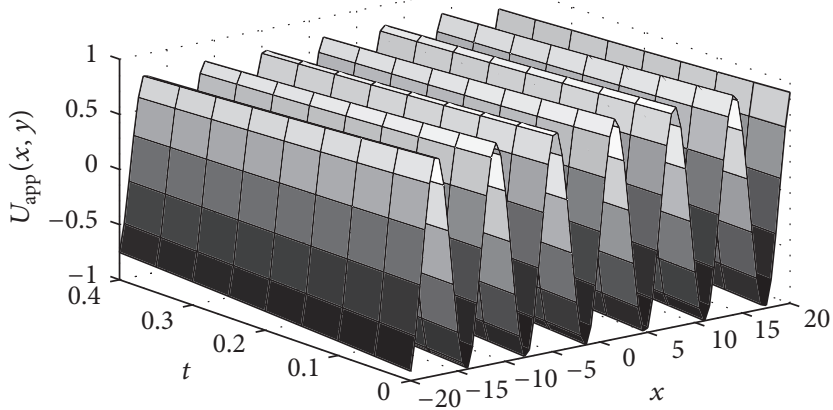

(a) The third-order approximate solution of $U(x, t)$

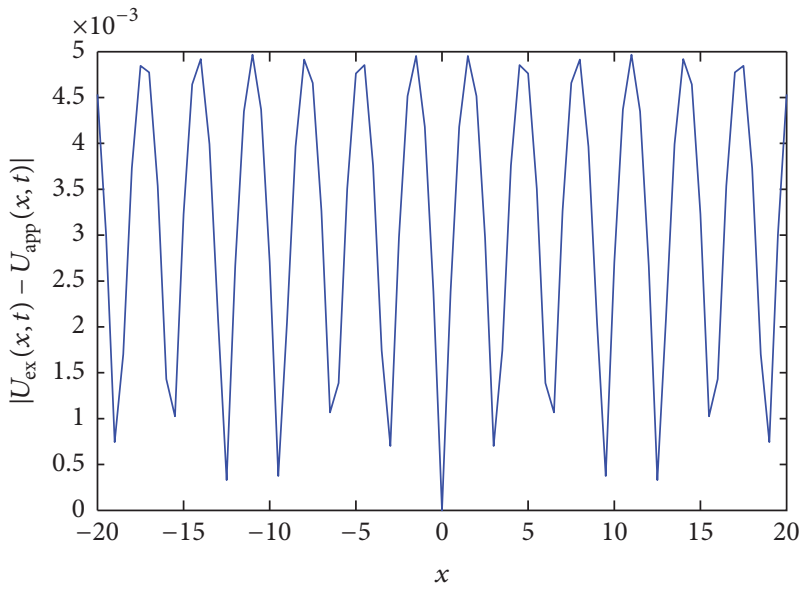

(c) The error $\left|U_{\text {app }}-U_{\text {ex }}\right|$ of $U(x, t)$ at $t=0.01$

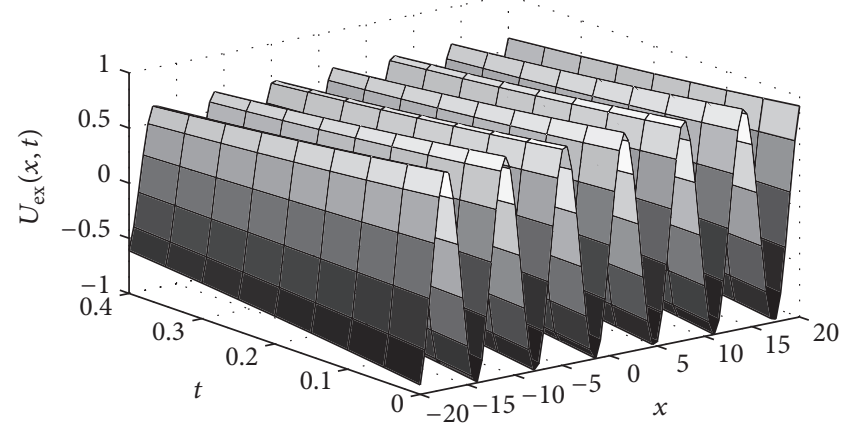

(b) The exact solution of $U(x, t)$

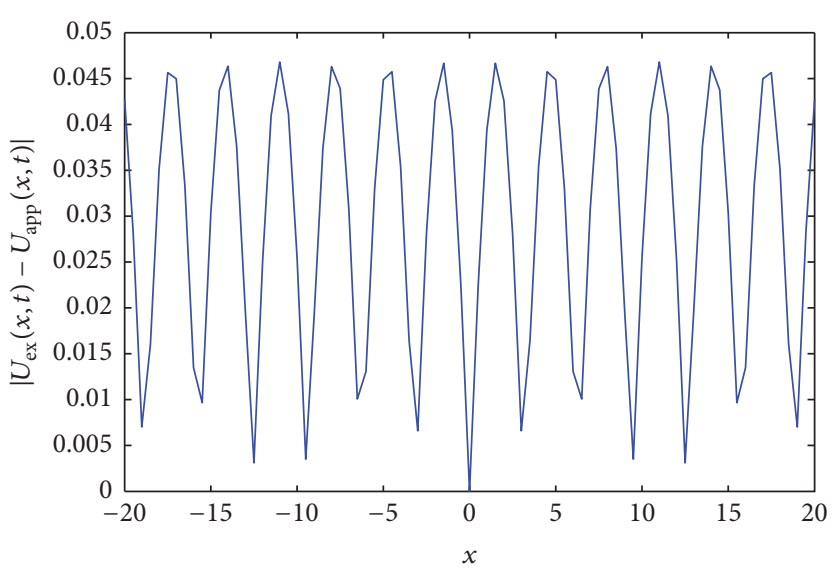

(d) The error $\left|U_{\text {app }}-U_{\text {ex }}\right|$ of $U(x, t)$ at $t=0.1$

Figure 1

Using HPSTM, when $\alpha=\gamma=\beta=\delta=1$, the third approximate solution of (23) is

$$
\begin{aligned}
U_{\text {app }}(x, t)= & \sin x+\frac{t}{2 !}(-\sin x)+\frac{t^{2}}{3 !}(\sin x) \\
& +\frac{t^{3}}{4 !}(-\sin x) \\
= & \sin x\left\{1+\frac{-t}{2 !}+\frac{(-t)^{2}}{3 !}+\frac{-t^{3}}{4 !}\right\}, \\
V_{\text {app }}(x, t)= & \sin x+\frac{t}{2 !}(-\sin x)+\frac{t^{2}}{3 !}(\sin x) \\
& +\frac{t^{3}}{4 !}(-\sin x) \\
= & \sin x\left\{1+\frac{-t}{2 !}+\frac{(-t)^{2}}{3 !}+\frac{-t^{3}}{4 !}\right\} .
\end{aligned}
$$

In general, the limit of the approximate solution is

$$
\begin{aligned}
U(x, t)= & \sin x+\frac{t}{2 !}(-\sin x)+\frac{t^{2}}{3 !}(\sin x) \\
& +\frac{t^{3}}{4 !}(-\sin x)+\cdots
\end{aligned}
$$

$$
\begin{aligned}
= & \sin x\left\{1+\frac{-t}{2 !}+\frac{(-t)^{2}}{3 !}+\frac{-t^{3}}{4 !}+\cdots\right\} \\
= & e^{-t} \sin x, \\
V(x, t)= & \sin x+\frac{t}{2 !}(-\sin x)+\frac{t^{2}}{3 !}(\sin x) \\
& +\frac{t^{3}}{4 !}(-\sin x)+\cdots \\
= & \sin x\left\{1+\frac{-t}{2 !}+\frac{(-t)^{2}}{3 !}+\frac{-t^{3}}{4 !}+\cdots\right\} \\
= & e^{-t} \sin x,
\end{aligned}
$$

which is as same as the exact solution. However, if the initial values are too complex to find the limit of the approximated solution, then we replace the exact solution by the approximated solution within a certain scale, which is useful in the application of engineering.

Thus we plot the images of the approximate solution (see Figure 1(a)), the exact solution (see Figure 1(b)), and the error function (see Figures 1(c) and 1(d)). It is clear that the error function $\left|U_{\text {app }}-U_{\text {ex }}\right|$ depends on time $t$. When time $t$ is small (e.g., $t=0.01$ ), the error function is in the scale of $10^{-3}$ (see 
Figure 1(c)), which indicates that this is a good approximation in the neighbour of time 0 for system (23) with some explicit parameters. However, when time becomes large (e.g., $t=0.1$ ), the error function tends to be large as well (see Figure 1(d)); that is to say, this method is only suitable for constructing the approximated solution around the initial data.

4.2. The Time-Space Fractional Coupled KdV System of Generalized Hirota-Satsuma Type. In this subsection, consider the time-space fractional generalization of the Hirota-Satsuma coupled KdV system

$$
\begin{array}{r}
D_{t}^{\alpha} U-\frac{1}{2} D_{x}^{3 \beta} U+3 U D_{x}^{\gamma} U-3 D_{x}^{\delta}(V W)=0 \\
D_{t}^{\alpha} V+D_{x}^{3 \lambda} V-3 U D_{x}^{\tau} V=0, \\
D_{t}^{\alpha} W+D_{x}^{3 \theta} W-3 U D_{x}^{\sigma} W=0,
\end{array}
$$

with respect to the initial conditions

$$
\begin{gathered}
U(x, 0)=a_{0}(x), \\
V(x, 0)=b_{0}(x), \\
W(x, 0)=c_{0}(x),
\end{gathered}
$$

where $0<\alpha, \gamma, \delta, \lambda, \tau \leq 1,2 / 3<\beta, \sigma, \theta \leq 1, U=U(x, t)$, $V=V(x, t), W=W(x, t),(x, t) \in \mathbb{R} \times[0, \infty)$. The HirotaSatsuma coupled KdV equation describes the unidirectional propagation of shallow water waves, which was initiated by Wu et al. [35]. Further (38) becomes a generalized fractional $\mathrm{KdV}$ equation for $U=0$ and a fractional $\mathrm{MKdV}$ equation for $V=0$.

Applying the Sumudu transform on both sides of (38) with the initial conditions, we obtain

$$
\begin{aligned}
& S[U(x, t)]=a_{0}(x)+u^{\alpha}\left(S \left[\frac{1}{2} D_{x}^{3 \beta} U(x, t)\right.\right. \\
& \left.\left.\quad-3 U(x, t) D_{x}^{\gamma} U(x, t)+3 D_{x}^{\delta}(V(x, t) W(x, t))\right]\right) \\
& S[V(x, t)]=b_{0}(x)-v^{\alpha}\left(S \left[D_{x}^{3 \lambda} V(x, t)\right.\right. \\
& \left.\left.\quad-3 U(x, t) D_{x}^{\tau} V(x, t)\right]\right) \\
& S[W(x, t)]=c_{0}(x)-w^{\alpha}\left(S \left[D_{x}^{3 \theta} W(x, t)\right.\right. \\
& \left.\left.\quad-3 U(x, t) D_{x}^{\sigma} W(x, t)\right]\right) .
\end{aligned}
$$

The inverse Sumudu transform of (40) implies that

$$
\begin{aligned}
& U(x, t)=a_{0}(x)+S^{-1}\left[u ^ { \alpha } \left(S \left[\frac{1}{2} D_{x}^{3 \beta} U(x, t)\right.\right.\right. \\
& -3 U(x, t) D_{x}^{\gamma} U(x, t) \\
& \left.\left.\left.+3 D_{x}^{\delta}(V(x, t) W(x, t))\right]\right)\right], \\
& V(x, t)=b_{0}(x)-S^{-1}\left[v ^ { \alpha } \left(S \left[D_{x}^{3 \lambda} V(x, t)\right.\right.\right. \\
& \left.\left.\left.-3 U(x, t) D_{x}^{\tau} V(x, t)\right]\right)\right],
\end{aligned}
$$

$$
\begin{aligned}
& W(x, t)=c_{0}(x)-S^{-1}\left[w ^ { \alpha } \left(S \left[D_{x}^{3 \theta} W(x, t)\right.\right.\right. \\
& \left.\left.\left.-3 U(x, t) D_{x}^{\sigma} W(x, t)\right]\right)\right]
\end{aligned}
$$

Via the homotopy perturbation method, it gives

$$
\begin{aligned}
& \sum_{n=0}^{\infty} p^{n} U_{n}(x, t)=a_{0}(x)+p \\
& \times S^{-1}\left[u ^ { \alpha } S \left[\frac{1}{2} D_{x}^{3 \beta}\left(\sum_{n=0}^{\infty} p^{n} U_{n}(x, t)\right)\right.\right. \\
& \left.\left.+\sum_{n=0}^{\infty} p^{n} H_{n}^{U}(x, t)\right]\right] \text {, } \\
& \sum_{n=0}^{\infty} p^{n} V_{n}(x, t)=b_{0}(x)+p \\
& \times S^{-1}\left[v ^ { \alpha } S \left[-D_{x}^{3 \lambda}\left(\sum_{n=0}^{\infty} p^{n} V_{n}(x, t)\right)\right.\right. \\
& \left.\left.+\sum_{n=0}^{\infty} p^{n} H_{n}^{V}(x, t)\right]\right] \text {, } \\
& \sum_{n=0}^{\infty} p^{n} W_{n}(x, t)=c_{0}(x)+p \\
& \times S^{-1}\left[w ^ { \alpha } S \left[-D_{x}^{3 \theta}\left(\sum_{n=0}^{\infty} W_{n}(x, t)\right)\right.\right. \\
& \left.\left.+\sum_{n=0}^{\infty} p^{n} H_{n}^{W}(x, t)\right]\right]
\end{aligned}
$$

where $H_{n}^{U}(x, t), H_{n}^{V}(x, t)$, and $H_{n}^{W}(x, t)$ are polynomials which denote the nonlinear term, and they are given by

$$
\begin{aligned}
\sum_{n=0}^{\infty} p^{n} H_{n}^{U}(x, t)= & 3 D_{x}^{\delta}(V(x, t) W(x, t)) \\
& -3 U(x, t) D_{x}^{\gamma} U(x, t), \\
\sum_{n=0}^{\infty} p^{n} H_{n}^{V}(x, t)= & 3 U(x, t) D_{x}^{\tau} V(x, t), \\
\sum_{n=0}^{\infty} p^{n} H_{n}^{W}(x, t)= & 3 U(x, t) D_{x}^{\sigma} W(x, t) .
\end{aligned}
$$

Set

$$
\begin{aligned}
& U_{n}(x, t):=\widetilde{U}_{n}(x) \frac{t^{n \alpha}}{\Gamma(n \alpha+1)}, \\
& V_{n}(x, t):=\widetilde{V}_{n}(x) \frac{t^{n \alpha}}{\Gamma(n \alpha+1)}, \\
& W_{n}(x, t):=\widetilde{W}_{n}(x) \frac{t^{n \alpha}}{\Gamma(n \alpha+1)},
\end{aligned}
$$


and then

$$
\begin{aligned}
H_{n}^{U} & =3 D_{x}^{\delta}\left(\sum_{i=0}^{n} \widetilde{V}_{i}(x) \widetilde{W}_{n-i}(x)\right. \\
\cdot & \left.\frac{t^{n \alpha}}{\Gamma(i \alpha+1) \Gamma((n-i) \alpha+1)}\right)-3 \sum_{i=0}^{n} \widetilde{U}_{i}(x) \\
\cdot & D_{x}^{\gamma} \widetilde{U}_{n-i}(x) \frac{t^{n \alpha}}{\Gamma(i \alpha+1) \Gamma((n-i) \alpha+1)}, \\
H_{n}^{V} & =3 \sum_{i=0}^{n} \widetilde{U}_{i}(x) D_{x}^{\tau} \widetilde{V}_{n-i}(x)
\end{aligned}
$$

$$
\begin{gathered}
\cdot \frac{t^{n \alpha}}{\Gamma(i \alpha+1) \Gamma((n-i) \alpha+1)}, \\
H_{n}^{W}=3 \sum_{i=0}^{n} \widetilde{U}_{i}(x) D_{x}^{\sigma} \widetilde{W}_{n-i}(x) \\
\cdot \frac{t^{n \alpha}}{\Gamma(i \alpha+1) \Gamma((n-i) \alpha+1)} .
\end{gathered}
$$

Comparing the coefficients of $p$ shows

$$
\begin{aligned}
p^{0}: \quad U_{0}(x, t)=\widetilde{U}_{0}(x)=a_{0}(x), \\
V_{0}(x, t)=\widetilde{V}_{0}(x)=b_{0}(x), \\
W_{0}(x, t)=\widetilde{W}_{0}(x)=c_{0}(x), \\
p^{1}: \quad U_{1}(x, t)=S^{-1}\left[u^{\alpha} S\left[\frac{1}{2} D_{x}^{3 \beta} U_{0}(x, t)+H_{0}^{U}(x, t)\right]\right] \\
=S^{-1}\left[u^{\alpha} S\left[\frac{1}{2} D_{x}^{3 \beta} \widetilde{U}_{0}(x)+3 D_{x}^{\delta}\left(\widetilde{V}_{0}(x) \widetilde{W}_{0}(x)\right)-3 \widetilde{U}_{0}(x) D_{x}^{\gamma} \widetilde{U}_{0}(x)\right]\right] \\
=\frac{t^{\alpha}}{\Gamma(\alpha+1)}\left(\frac{1}{2} D_{x}^{3 \beta} \widetilde{U}_{0}(x)+3 D_{x}^{\delta}\left(\widetilde{V}_{0}(x) \widetilde{W}_{0}(x)\right)-3 \widetilde{U}_{0}(x) D_{x}^{\gamma} \widetilde{U}_{0}(x)\right)=\frac{t^{\alpha}}{\Gamma(\alpha+1)} \widetilde{U}_{1}(x), \\
\quad V_{1}(x, t)=S^{-1}\left[v^{\alpha} S\left[-D_{x}^{3 \lambda} V_{0}(x, t)+H_{0}^{V}(x, t)\right]\right]=S^{-1}\left[v^{\alpha} S\left[-D_{x}^{3 \lambda} \widetilde{V}_{0}(x)+3 \widetilde{U}_{0}(x) D_{x}^{\tau} \widetilde{V}_{0}(x)\right]\right] \\
=\frac{t^{\alpha}}{\Gamma(\alpha+1)}\left(-D_{x}^{3 \lambda} \widetilde{V}_{0}(x)+3 \widetilde{U}_{0}(x) D_{x}^{\gamma} \widetilde{U}_{0}(x)\right)=\frac{t^{\alpha}}{\Gamma(\alpha+1)} \widetilde{V}_{1}(x), \\
\quad W_{1}(x, t)=S^{-1}\left[w^{\alpha} S\left[-D_{x}^{3 \theta} W_{0}(x, t)+H_{0}^{W}(x, t)\right]\right]=S^{-1}\left[w^{\alpha} S\left[-D_{x}^{3 \theta} \widetilde{W}_{0}(x)+3 \widetilde{U}_{0}(x) D_{x}^{\sigma} \widetilde{W}_{0}(x)\right]\right] \\
=\frac{t^{\alpha}}{\Gamma(\alpha+1)}\left(-D_{x}^{3 \theta} \widetilde{W}_{0}(x)+3 \widetilde{U}_{0}(x) D_{x}^{\sigma} \widetilde{W}_{0}(x)\right)=\frac{t^{\alpha}}{\Gamma(\alpha+1)} \widetilde{W}_{1}(x) .
\end{aligned}
$$

Generally, one has

$p^{k}: \quad U_{k}(x, t)$

$$
\begin{aligned}
& =\frac{t^{k \alpha}}{\Gamma(k \alpha+1)}\left\{\frac{1}{2} D_{x}^{3 \beta} \widetilde{U}_{k-1}(x)+\sum_{i=0}^{k-1} \frac{\Gamma((k-1) \alpha+1)}{\Gamma(i \alpha+1) \Gamma((k-i-1) \alpha+1)}\left(3 D_{x}^{\delta}\left(\widetilde{V}_{k-1-i}(x) \widetilde{W}_{i}(x)\right)-3 \widetilde{U}_{i}(x) D_{x}^{\gamma} \widetilde{U}_{k-1-i}(x)\right)\right\} \\
& =\frac{t^{k \alpha}}{\Gamma(k \alpha+1)} \widetilde{U}_{k}(x), \\
& V_{k}(x, t)=\frac{t^{k \alpha}}{\Gamma(k \alpha+1)}\left\{-D_{x}^{3 \lambda} \widetilde{V}_{k-1}(x)+\sum_{i=0}^{k-1} \frac{\Gamma((k-1) \alpha+1)}{\Gamma(i \alpha+1) \Gamma((k-i-1) \alpha+1)} 3 \widetilde{U}_{i}(x) D_{x}^{\tau} \widetilde{V}_{k-1-i}(x)\right\}=\frac{t^{k \alpha}}{\Gamma(k \alpha+1)} \widetilde{V}_{k}(x), \\
& W_{k}(x, t)=\frac{t^{k \alpha}}{\Gamma(k \alpha+1)}\left\{-D_{x}^{3 \theta} \widetilde{W}_{k-1}(x)+\sum_{i=0}^{k-1} \frac{\Gamma((k-1) \alpha+1)}{\Gamma(i \alpha+1) \Gamma((k-i-1) \alpha+1)} 3 \widetilde{U}_{i}(x) D_{x}^{\sigma} \widetilde{W}_{k-1-i}(x)\right\} \\
& =\frac{t^{k \alpha}}{\Gamma(k \alpha+1)} \widetilde{W}_{k}(x),
\end{aligned}
$$


where

$$
\begin{aligned}
& \widetilde{U}_{k}(x)=\frac{1}{2} D_{x}^{3 \beta} \widetilde{U}_{k-1}(x) \\
& +\sum_{i=0}^{k-1} \frac{\Gamma((k-1) \alpha+1)}{\Gamma(i \alpha+1) \Gamma((k-i-1) \alpha+1)} \\
& \cdot\left(3 D_{x}^{\delta}\left(\widetilde{V}_{k-1-i}(x) \widetilde{W}_{i}(x)\right)\right. \\
& \left.-3 \widetilde{U}_{i}(x) D_{x}^{\gamma} \widetilde{U}_{k-1-i}(x)\right), \\
& \widetilde{V}_{k}(x)=-D_{x}^{3 \lambda} \widetilde{V}_{k-1}(x) \\
& +\sum_{i=0}^{k-1} \frac{\Gamma((k-1) \alpha+1)}{\Gamma(i \alpha+1) \Gamma((k-i-1) \alpha+1)} 3 \widetilde{U}_{i}(x) \\
& \cdot D_{x}^{\tau} \widetilde{V}_{k-1-i}(x), \\
& \widetilde{W}_{k}(x)=-D_{x}^{3 \theta} \widetilde{W}_{k-1}(x) \\
& +\sum_{i=0}^{k-1} \frac{\Gamma((k-1) \alpha+1)}{\Gamma(i \alpha+1) \Gamma((k-i-1) \alpha+1)} 3 \widetilde{U}_{i}(x) \\
& +D_{x}^{\sigma} \widetilde{W}_{k-1-i}(x) .
\end{aligned}
$$

Therefore, the approximate series solution of (38) is

$$
\begin{aligned}
& U(x, t)=\sum_{k=0}^{\infty} U_{k}(x, t)=\sum_{k=0}^{\infty} \widetilde{U}_{k}(x) \frac{t^{k \alpha}}{\Gamma(k \alpha+1)}, \\
& V(x, t)=\sum_{k=0}^{\infty} V_{k}(x, t)=\sum_{k=0}^{\infty} \widetilde{V}_{k}(x) \frac{t^{k \alpha}}{\Gamma(k \alpha+1)}, \\
& W(x, t)=\sum_{k=0}^{\infty} W_{k}(x, t)=\sum_{k=0}^{\infty} \widetilde{W}_{k}(x) \frac{t^{k \alpha}}{\Gamma(k \alpha+1)} .
\end{aligned}
$$

Particularly, when $\alpha=\gamma=\delta=\lambda=1, \tau=\beta=\sigma=\theta=2 / 3$, and the special initial value of (38) is

$$
\begin{aligned}
& U(x, 0)=-2+4 \tanh ^{2} x, \\
& V(x, 0)=-4+4 \tanh ^{2} x, \\
& W(x, 0)=1+\tanh ^{2} x,
\end{aligned}
$$

then the exact solutions are

$$
\begin{gathered}
U(x, t)=-2+4 \tanh ^{2}(x+2 t), \\
V(x, t)=-4+4 \tanh ^{2}(x+2 t), \\
W(x, t)=1+\tanh ^{2}(x+2 t) .
\end{gathered}
$$

Under these special conditions, via HPSTM, the first approximate solution of (38) is

$$
\begin{aligned}
& U_{\text {app }}(x, t)=-2+4 \tanh ^{2} x+16\left(\tanh x-\tanh ^{3} x\right) t, \\
& V_{\text {app }}(x, t)=-4+4 \tanh ^{2} x+16\left(\tanh x-\tanh ^{3} x\right) t, \\
& W_{\text {app }}(x, t)=1+\tanh ^{2} x+4\left(\tanh x-\tanh ^{3} x\right) t
\end{aligned}
$$

Similarly, we obtain the following numerical results: see Figures 2(a), 2(b), 2(c), 2(d), 3(a), 3(b), 3(c), 3(d), 4(a), 4(b), $4(\mathrm{c})$, and $4(\mathrm{~d})$.

4.3. The Time-Space Fractional Coupled Shallow Water System. Shallow water systems are widely used in many areas of fluid dynamics, such as multiphase flows [36], turbulence [37], and viscoelasticity [38]. It is well known that the shallow water systems can accurately predict both the hydraulic parameters under conditions of slow erosion and low sediment concentration. Let us consider the time-space fractional coupled shallow water system

$$
\begin{array}{r}
D_{t}^{\alpha} U+U D_{x}^{\beta} U+D_{x}^{\gamma} V+a D_{x}^{2 \delta} U=0, \\
D_{t}^{\alpha} V+V D_{x}^{\lambda} U+U D_{x}^{\tau} V-a D_{x}^{2 \theta} V+b D_{x}^{3 \sigma} U=0
\end{array}
$$

with initial values

$$
\begin{aligned}
& U(x, 0)=a_{0}(x), \\
& V(x, 0)=b_{0}(x),
\end{aligned}
$$

where $0<\alpha, \beta, \gamma, \lambda, \tau \leq 1,1 / 2<\delta, \theta \leq 1,2 / 3<\sigma \leq 1$, $U=U(x, t), V=V(x, t),(x, t) \in \mathbb{R} \times[0,1 / 3]$.

Applying the Sumudu transform on both sides of (53) with the initial conditions, we obtain

$$
\begin{aligned}
& S[U(x, t)]=a_{0}(x)+u^{\alpha}\left(S \left[-U(x, t) D_{x}^{\beta} U(x, t)\right.\right. \\
& \left.\left.\quad-D_{x}^{\gamma} V(x, t)-a D_{x}^{2 \delta} U(x, t)\right]\right), \\
& S[V(x, t)]=b_{0}(x)+v^{\alpha}\left(S \left[-V(x, t) D_{x}^{\lambda} U(x, t)\right.\right. \\
& \quad-U(x, t) D_{x}^{\tau} V(x, t)+a D_{x}^{2 \theta} V(x, t) \\
& \left.\left.\quad-b D_{x}^{3 \sigma} U(x, t)\right]\right) .
\end{aligned}
$$

The inverse Sumudu transform of (55) implies that

$$
\begin{aligned}
& U(x, t)=a_{0}(x)+S^{-1}\left[u ^ { \alpha } \left(S \left[-U(x, t) D_{x}^{\beta} U(x, t)\right.\right.\right. \\
& \left.\left.\left.\quad-D_{x}^{\gamma} V(x, t)-a D_{x}^{2 \delta} U(x, t)\right]\right)\right], \\
& V(x, t)=b_{0}(x)+S^{-1}\left[v ^ { \alpha } \left(S \left[-V(x, t) D_{x}^{\lambda} U(x, t)\right.\right.\right. \\
& \quad-U(x, t) D_{x}^{\tau} V(x, t)+a D_{x}^{2 \theta} V(x, t) \\
& \left.\left.\left.-b D_{x}^{3 \sigma} U(x, t)\right]\right)\right] .
\end{aligned}
$$

According to homotopy perturbation method, we have

$$
\begin{aligned}
& \sum_{n=0}^{\infty} p^{n} U_{n}(x, t)=a_{0}(x)+p \\
& \quad \times S^{-1}\left[u ^ { \alpha } S \left[-D_{x}^{\gamma}\left(\sum_{n=0}^{\infty} p^{n} V_{n}(x, t)\right)\right.\right.
\end{aligned}
$$




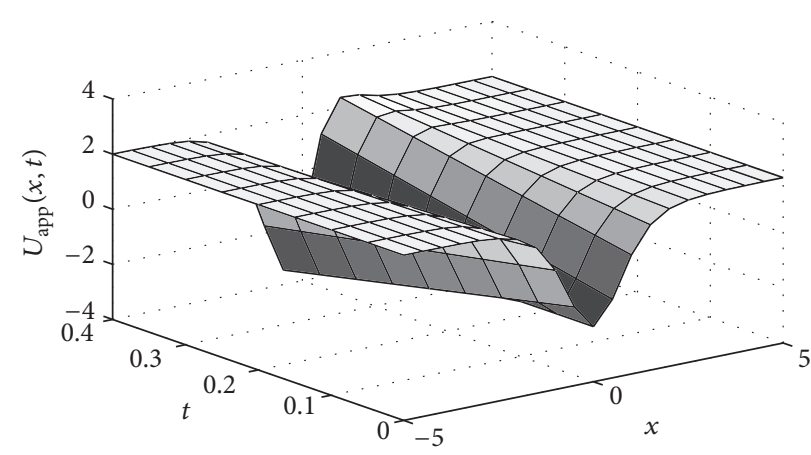

(a) The first-order approximate solution of $U(x, t)$

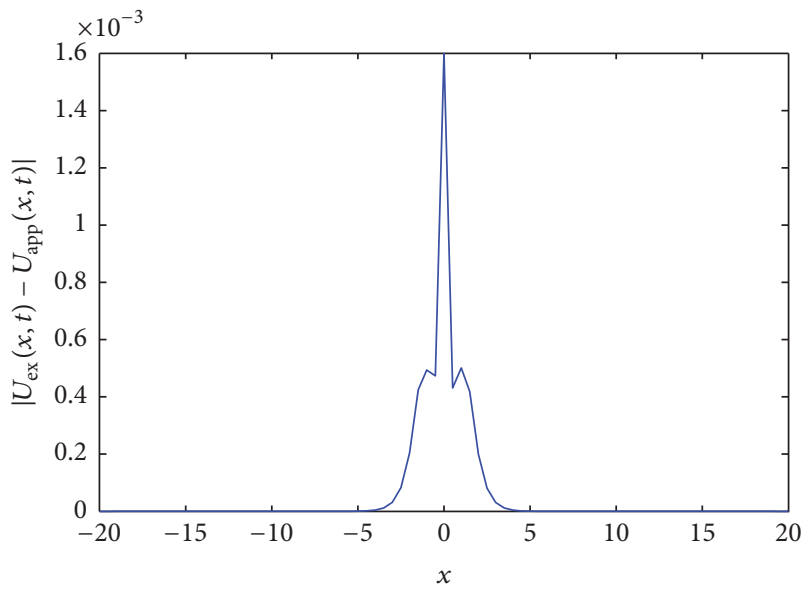

(c) The error $\left|U_{\text {app }}-U_{\text {ex }}\right|$ of $U(x, t)$ at $t=0.01$

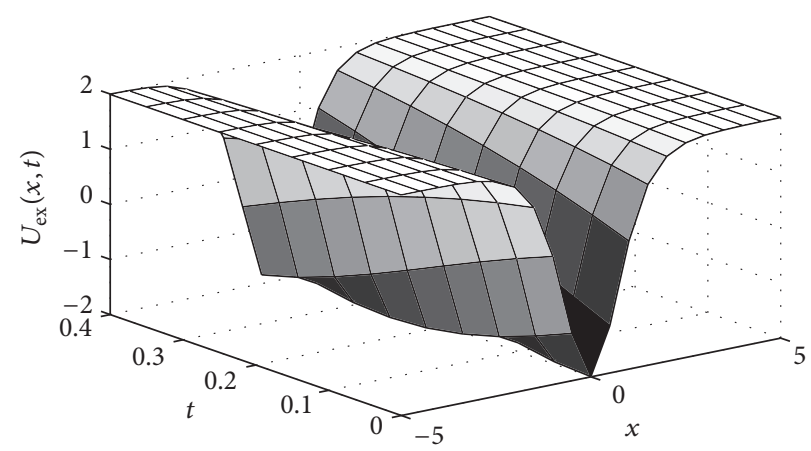

(b) The exact solution of $U(x, t)$

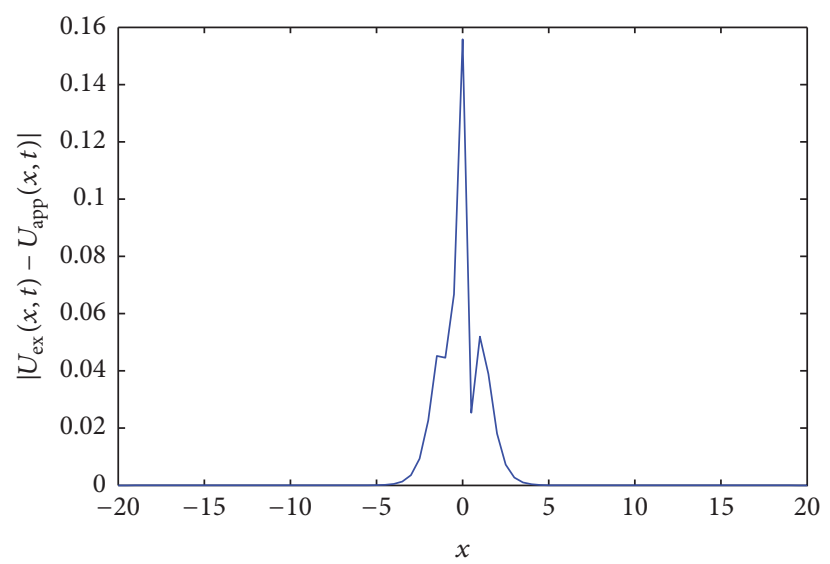

(d) The error $\left|U_{\text {app }}-U_{\text {ex }}\right|$ of $U(x, t)$ at $t=0.1$

Figure 2

$$
\begin{aligned}
& \left.\left.-a D_{x}^{2 \delta}\left(\sum_{n=0}^{\infty} p^{n} U_{n}(x, t)\right)-\sum_{n=0}^{\infty} p^{n} H_{n}^{U}(x, t)\right]\right], \\
& \sum_{n=0}^{\infty} p^{n} V_{n}(x, t)=b_{0}(x)+p \\
& \quad \times S^{-1}\left[v ^ { \alpha } S \left[a D_{x}^{2 \theta}\left(\sum_{n=0}^{\infty} p^{n} V_{n}(x, t)\right)\right.\right. \\
& \left.\left.-b D_{x}^{3 \sigma}\left(\sum_{n=0}^{\infty} p^{n} U_{n}(x, t)\right)-\sum_{n=0}^{\infty} p^{n} H_{n}^{V}(x, t)\right]\right]
\end{aligned}
$$

where $H_{n}^{U}(x, t)$ and $H_{n}^{V}(x, t)$ are polynomials of the nonlinear term and are given by

$$
\begin{aligned}
\sum_{n=0}^{\infty} p^{n} H_{n}^{U}(x, t)= & U(x, t) D_{x}^{\beta} U(x, t), \\
\sum_{n=0}^{\infty} p^{n} H_{n}^{V}(x, t)= & V(x, t) D_{x}^{\lambda} U(x, t) \\
& +U(x, t) D_{x}^{\tau} V(x, t) .
\end{aligned}
$$

Setting

$$
\begin{aligned}
& U_{n}(x, t):=\widetilde{U}_{n}(x) \frac{t^{n \alpha}}{\Gamma(n \alpha+1)}, \\
& V_{n}(x, t):=\widetilde{V}_{n}(x) \frac{t^{n \alpha}}{\Gamma(n \alpha+1)},
\end{aligned}
$$

then we arrive at

$$
\begin{aligned}
& H_{n}^{U}(x, t) \\
& \quad=\sum_{i=0}^{n} \widetilde{U}_{i}(x) D_{x}^{\beta} \widetilde{U}_{n-i}(x) \frac{t^{n \alpha}}{\Gamma(i \alpha+1) \Gamma((n-i) \alpha+1)}, \\
& H_{n}^{V}(x, t) \\
& \quad=\left(\sum_{i=0}^{n} \widetilde{V}_{i}(x) D_{x}^{\lambda} \widetilde{U}_{n-i}(x)+\sum_{i=0}^{n} \widetilde{U}_{i}(x) D_{x}^{\tau} \widetilde{V}_{n-i}(x)\right) \\
& \quad \frac{t^{n \alpha}}{\Gamma(i \alpha+1) \Gamma((n-i) \alpha+1)} .
\end{aligned}
$$

Comparing the coefficients of $p$ yields 


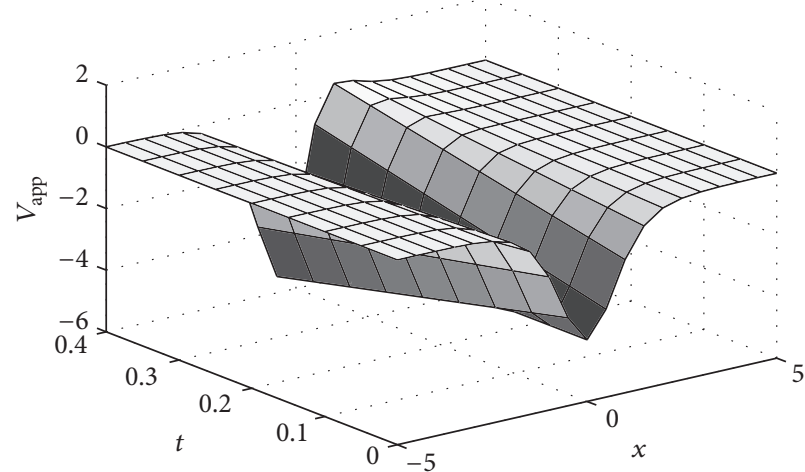

(a) The first-order approximate solution of $V(x, t)$

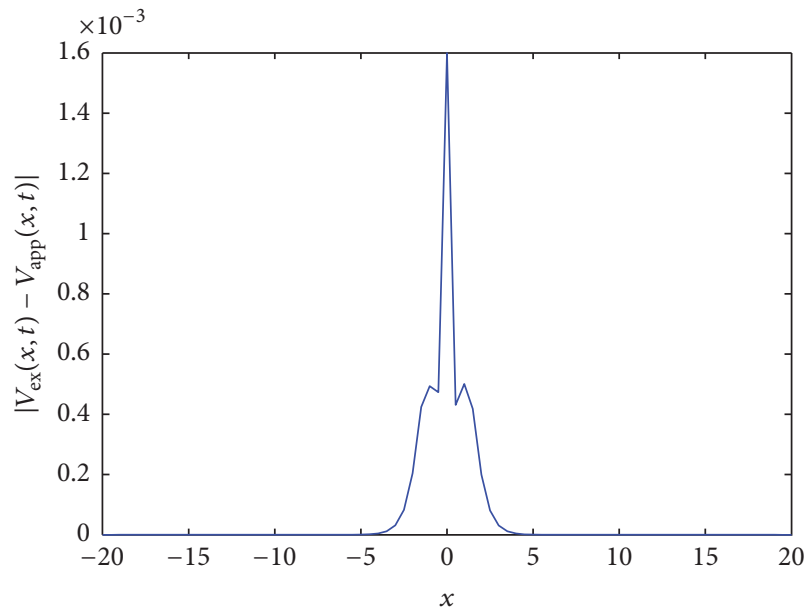

(c) The error $\left|V_{\text {app }}-V_{\text {ex }}\right|$ of $V(x, t)$ at $t=0.01$

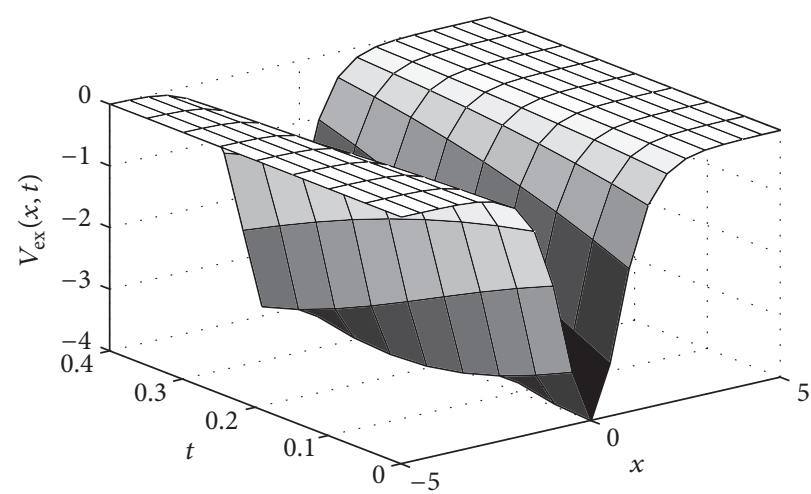

(b) The exact solution of $V(x, t)$

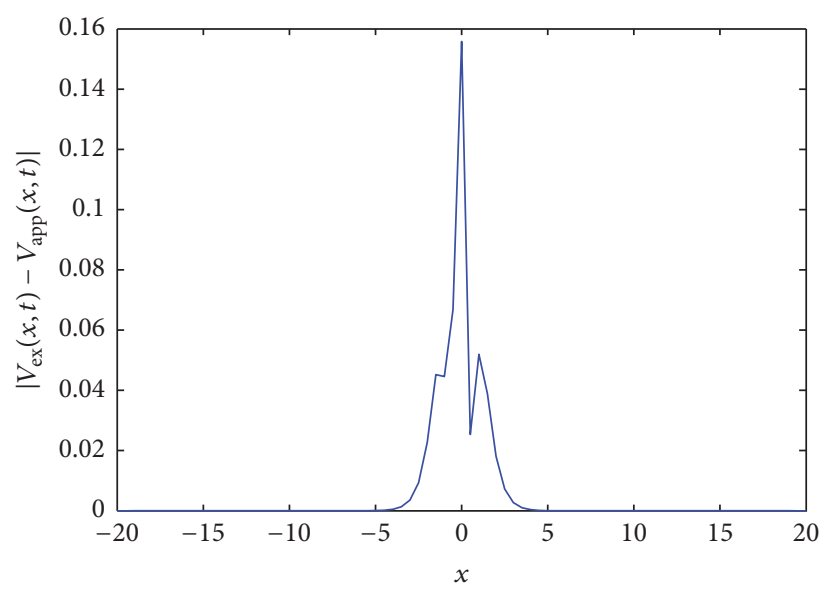

(d) The error $\left|V_{\text {app }}-V_{\text {ex }}\right|$ of $V(x, t)$ at $t=0.1$

Figure 3

$$
\begin{aligned}
p^{0}: & U_{0}(x, t)=\widetilde{U}_{0}(x)=a_{0}(x) \\
& V_{0}(x, t)=\widetilde{V}_{0}(x)=b_{0}(x) \\
p^{1}: & U_{1}(x, t)=S^{-1}\left[u^{\alpha} S\left[-D_{x}^{\gamma} V_{0}(x, t)-a D_{x}^{2 \delta} U_{0}(x, t)-H_{0}^{U}(x)\right]\right] \\
& =S^{-1}\left[u^{\alpha} S\left[-D_{x}^{\gamma} \widetilde{U}_{0}(x)-a D_{x}^{2 \delta} \widetilde{U}_{0}(x)-\widetilde{U}_{0}(x) D_{x}^{\beta} \widetilde{U}_{0}(x)\right]\right] \\
& =\frac{t^{\alpha}}{\Gamma(\alpha+1)}\left(-D_{x}^{\gamma} \widetilde{U}_{0}(x)-a D_{x}^{2 \delta} \widetilde{U}_{0}(x)-\widetilde{U}_{0}(x) D_{x}^{\beta} \widetilde{U}_{0}(x)\right)=\frac{t^{\alpha}}{\Gamma(\alpha+1)} \widetilde{U}_{1}(x) \\
& V_{1}(x, t)=S^{-1}\left[v^{\alpha} S\left[-b D_{x}^{3 \sigma} V_{0}(x, t)+a D_{x}^{2 \theta} V_{0}(x, t)-H_{0}^{V}(x)\right]\right] \\
& =S^{-1}\left[v^{\alpha} S\left[-b D_{x}^{3 \sigma} \widetilde{V}_{0}(x)+a D_{x}^{2 \theta} \widetilde{V}_{0}(x)-\widetilde{V}_{0}(x) D_{x}^{\lambda} \widetilde{U}_{0}(x)-\widetilde{U}_{0}(x) D_{x}^{\tau} \widetilde{V}_{0}(x)\right]\right] \\
& =\frac{t^{\alpha}}{\Gamma(\alpha+1)}\left(-b D_{x}^{3 \sigma} \widetilde{V}_{0}(x)+a D_{x}^{2 \theta} \widetilde{V}_{0}(x)-\widetilde{V}_{0}(x) D_{x}^{\lambda} \widetilde{U}_{0}(x)-\widetilde{U}_{0}(x) D_{x}^{\tau} \widetilde{V}_{0}(x)\right)=\frac{t^{\alpha}}{\Gamma(\alpha+1)} \widetilde{V}_{1}(x) .
\end{aligned}
$$

Generally, we have

$$
\begin{aligned}
p^{k}: & U_{k}(x, t)=\frac{t^{k \alpha}}{\Gamma(k \alpha+1)}\left\{-D_{x}^{\gamma} \widetilde{V}_{k-1}(x)-a D_{x}^{2 \delta} \widetilde{U}_{k-1}(x)-\sum_{i=0}^{k-1} \frac{\Gamma((k-1) \alpha+1)}{\Gamma(i \alpha+1) \Gamma((k-i-1) \alpha+1)} \widetilde{U}_{i}(x) D_{x}^{\beta} \widetilde{U}_{k-1-i}(x)\right\} \\
& =\frac{t^{k \alpha}}{\Gamma(k \alpha+1)} \widetilde{U}_{k}(x),
\end{aligned}
$$




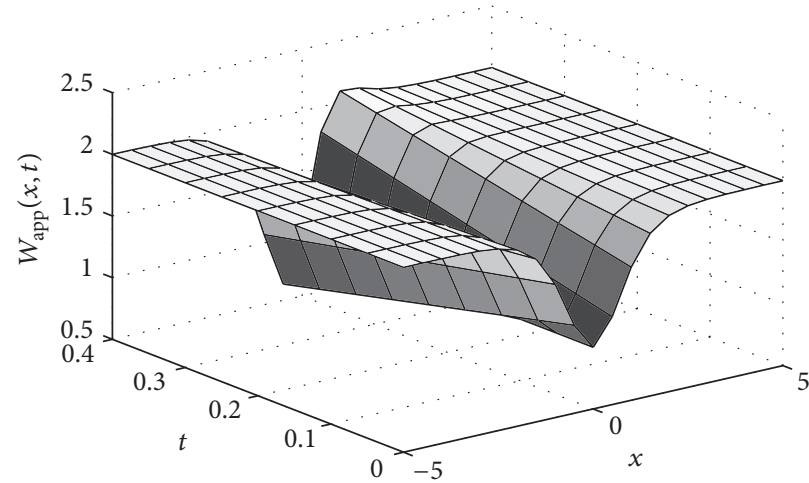

(a) The first-order approximate solution of $W(x, t)$

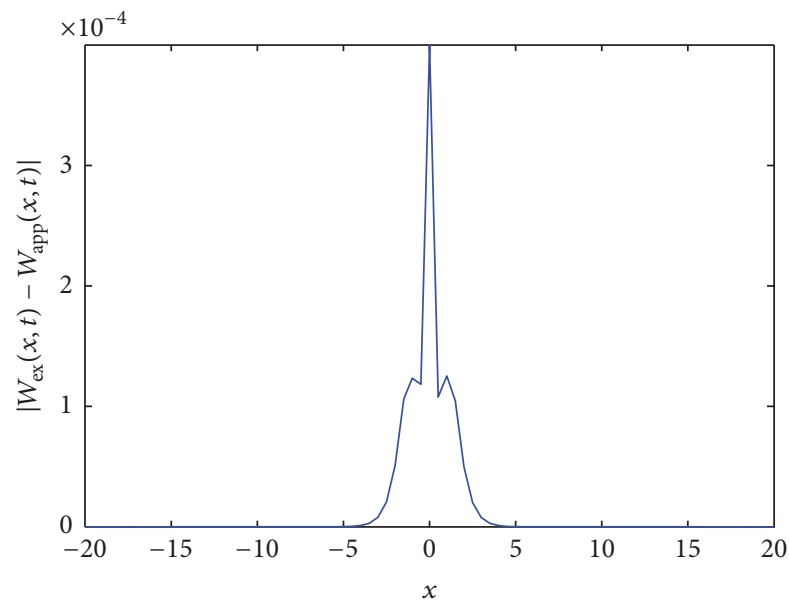

(c) The error $\left|W_{\text {app }}-W_{\text {ex }}\right|$ of $W(x, t)$ at $t=0.01$

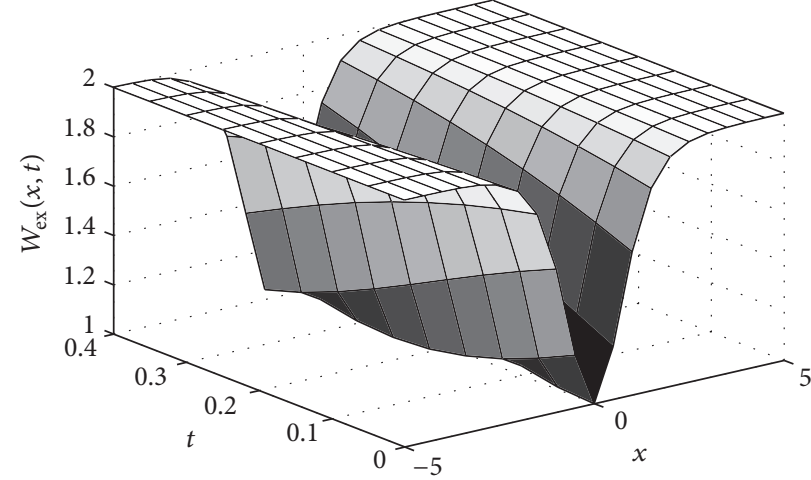

(b) The exact solution of $W(x, t)$

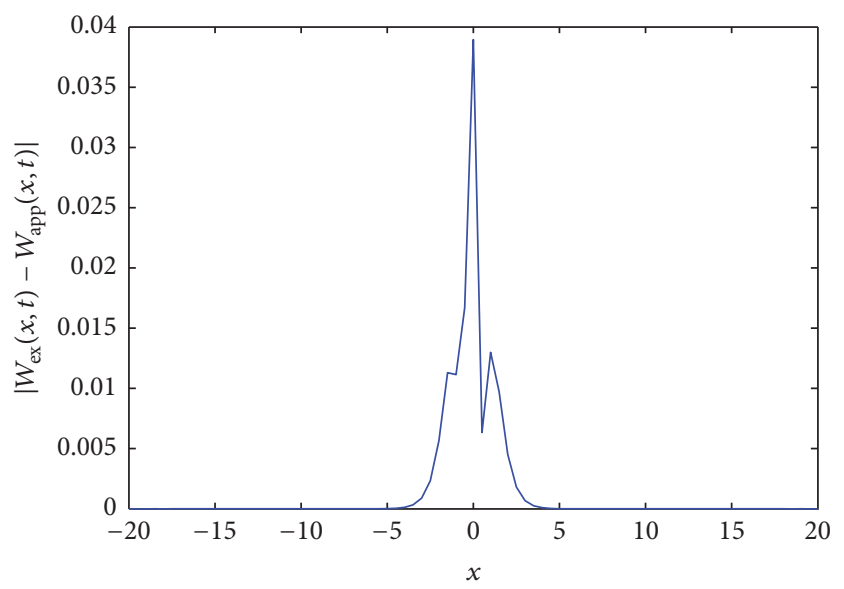

(d) The error $\left|W_{\text {app }}-W_{\text {ex }}\right|$ of $W(x, t)$ at $t=0.1$

FIGURE 4

$$
\begin{aligned}
& V_{k}(x, t) \\
& =\frac{t^{k \alpha}}{\Gamma(k \alpha+1)}\left\{-b D_{x}^{3 \sigma} \widetilde{V}_{k-1}(x)+a D_{x}^{2 \theta} \widetilde{V}_{k-1}(x)-\sum_{i=0}^{k-1} \frac{\Gamma((k-1) \alpha+1)}{\Gamma(i \alpha+1) \Gamma((k-i-1) \alpha+1)}\left(\widetilde{U}_{i}(x) D_{x}^{\lambda} \widetilde{V}_{k-1-i}(x)+\widetilde{V}_{i}(x) D_{x}^{\tau} \widetilde{U}_{k-1-i}(x)\right)\right\} \\
& =\frac{t^{k \alpha}}{\Gamma(k \alpha+1)} \widetilde{V}_{k}(x),
\end{aligned}
$$

where

$$
\begin{gathered}
\widetilde{U}_{k}(x)=-D_{x}^{\gamma} \widetilde{V}_{k-1}(x)-a D_{x}^{2 \delta} \widetilde{U}_{k-1}(x) \\
-\sum_{i=0}^{k-1} \frac{\Gamma((k-1) \alpha+1)}{\Gamma(i \alpha+1) \Gamma((k-i-1) \alpha+1)} \widetilde{U}_{i}(x) \\
\cdot D_{x}^{\beta} \widetilde{U}_{k-1-i}(x), \\
\widetilde{V}_{k}(x)=-b D_{x}^{3 \sigma} \widetilde{V}_{k-1}(x)+a D_{x}^{2 \theta} \widetilde{V}_{k-1}(x) \\
-\sum_{i=0}^{k-1} \frac{\Gamma((k-1) \alpha+1)}{\Gamma(i \alpha+1) \Gamma((k-i-1) \alpha+1)}\left(\widetilde{U}_{i}(x)\right. \\
\left.\cdot D_{x}^{\lambda} \widetilde{V}_{k-1-i}(x)+\widetilde{V}_{i}(x) D_{x}^{\tau} \widetilde{U}_{k-1-i}(x)\right) .
\end{gathered}
$$

Hence, the series solution is

$$
\begin{aligned}
& U(x, t)=\sum_{k=0}^{\infty} U_{k}(x, t)=\sum_{k=0}^{\infty} \widetilde{U}_{k}(x) \frac{t^{k \alpha}}{\Gamma(k \alpha+1)}, \\
& V(x, t)=\sum_{k=0}^{\infty} V_{k}(x, t)=\sum_{k=0}^{\infty} \widetilde{V}_{k}(x) \frac{t^{k \alpha}}{\Gamma(k \alpha+1)} .
\end{aligned}
$$

4.4. The Time-Space Fractional Coupled KdV System. KdV equation plays an important role in nonlinear equations for wide applications in physics and engineering. Hirota and Satsuma [39] firstly found coupled KdV system to describe the iterations of water waves; meanwhile, they claimed that the system exists with a soliton solution. In [40], Fan and 
Zhang settled several kinds of solutions by an improved homogeneous method. The time-space fractional coupled $\mathrm{KdV}$ equation is a generalization of the classical coupled $\mathrm{KdV}$ equation. In this subsection, we consider the following timespace fractional coupled $\mathrm{KdV}$ system:

$$
\begin{array}{r}
D_{t}^{\alpha} U-a D_{x}^{3 \beta} U-6 a U D_{x}^{\gamma} U-2 b V D_{x}^{\delta} V=0 \\
D_{t}^{\alpha} V+D_{x}^{3 \lambda} V+3 U D_{x}^{\tau} V=0
\end{array}
$$

with respect to initial values

$$
\begin{aligned}
& U(x, 0)=a_{0}(x), \\
& V(x, 0)=b_{0}(x),
\end{aligned}
$$

where $0<\alpha, \gamma, \delta, \tau \leq 1,2 / 3<\beta, \lambda \leq 1,(x, t) \in \mathbb{R} \times[0, \infty)$, and the coefficients $a, b$ are constants.

Applying the Sumudu transform on both sides of (65) with the initial conditions, we obtain

$$
\begin{aligned}
& S[U(x, t)]=a_{0}(x)+u^{\alpha}\left(S \left[a D_{x}^{3 \beta} U(x, t)\right.\right. \\
& \left.\left.\quad+6 a U(x, t) D_{x}^{\gamma} U(x, t)+2 b V(x, t) D_{x}^{\delta} V(x, t)\right]\right), \\
& S[V(x, t)]=b_{0}(x)-v^{\alpha}\left(S \left[D_{x}^{3 \lambda} V(x, t)\right.\right. \\
& \left.\left.\quad+3 U(x, t) D_{x}^{\tau} V(x, t)\right]\right) .
\end{aligned}
$$

The inverse Sumudu transform of (67) implies that

$$
\begin{aligned}
& U(x, t)=a_{0}(x)+S^{-1}\left[u ^ { \alpha } \left(S \left[a D_{x}^{3 \beta} U(x, t)\right.\right.\right. \\
& \left.\left.\left.\quad+6 a U(x, t) D_{x}^{\gamma} U(x, t)+2 b V(x, t) D_{x}^{\delta} V(x, t)\right]\right)\right] \\
& V(x, t)=b_{0}(x)-S^{-1}\left[v ^ { \alpha } \left(S \left[D_{x}^{3 \lambda} V(x, t)\right.\right.\right. \\
& \left.\left.\left.\quad+3 U(x, t) D_{x}^{\tau} V(x, t)\right]\right)\right] .
\end{aligned}
$$

Analogously, using homotopy perturbation method gives

$$
\begin{aligned}
& \sum_{n=0}^{\infty} p^{n} U_{n}(x, t)=a_{0}(x)+p \\
& \times S^{-1}\left[u ^ { \alpha } S \left[a D_{x}^{3 \beta}\left(\sum_{n=0}^{\infty} p^{n} U_{n}(x, t)\right)\right.\right. \\
& \left.\left.+\sum_{n=0}^{\infty} p^{n} H_{n}^{U}(x, t)\right]\right]
\end{aligned}
$$

$$
\begin{aligned}
& \sum_{n=0}^{\infty} p^{n} V_{n}(x, t)=b_{0}(x)+p \\
& \quad \times S^{-1}\left[v ^ { \alpha } S \left[-D_{x}^{3 \lambda}\left(\sum_{n=0}^{\infty} p^{n} V_{n}(x, t)\right)\right.\right. \\
& \left.\left.\quad+\sum_{n=0}^{\infty} p^{n} H_{n}^{V}(x, t)\right]\right]
\end{aligned}
$$

where $H_{n}^{U}(x, t)$ and $H_{n}^{V}(x, t)$ are homotopy polynomials coefficients of the nonlinear term, which are given by

$$
\begin{aligned}
\sum_{n=0}^{\infty} p^{n} H_{n}^{U}(x, t)= & 6 a U(x, t) D_{x}^{\gamma} U(x, t) \\
& +2 b V(x, t) D_{x}^{\delta} V(x, t) \\
\sum_{n=0}^{\infty} p^{n} H_{n}^{V}(x, t)= & -3 U(x, t) D_{x}^{\tau} V(x, t) .
\end{aligned}
$$

Setting

$$
\begin{aligned}
& U_{n}(x, t):=\widetilde{U}_{n}(x) \frac{t^{n \alpha}}{\Gamma(n \alpha+1)}, \\
& V_{n}(x, t):=\widetilde{V}_{n}(x) \frac{t^{n \alpha}}{\Gamma(n \alpha+1)},
\end{aligned}
$$

then

$$
\begin{aligned}
& H_{n}^{U}(x, t)=6 a \sum_{i=0}^{n} \widetilde{U}_{i}(x) D_{x}^{\gamma} \widetilde{U}_{n-i}(x) \\
& \cdot \frac{t^{n \alpha}}{\Gamma(i \alpha+1) \Gamma((n-i) \alpha+1)} \\
& \quad+2 b \sum_{i=0}^{n} \frac{t^{n \alpha}}{\Gamma(i \alpha+1) \Gamma((n-i) \alpha+1)} \widetilde{V}_{i}(x) \\
& \cdot D_{x}^{\delta} \widetilde{V}_{n-i}(x), \\
& H_{n}^{V}(x, t)=-3 \sum_{i=0}^{n} \widetilde{U}_{i}(x) D_{x}^{\tau} \widetilde{V}_{n-i}(x) \\
& \cdot \frac{t^{n \alpha}}{\Gamma(i \alpha+1) \Gamma((n-i) \alpha+1)} .
\end{aligned}
$$

$$
\begin{array}{ll}
p^{0}: \quad U_{0}(x, t)=\widetilde{U}_{0}(x)=a_{0}(x) \\
V_{0}(x, t)=\widetilde{V}_{0}(x)=b_{0}(x) \\
p^{1}: \quad U_{1}(x, t)=S^{-1}\left[u^{\alpha} S\left[a D_{x}^{3 \beta} U_{0}(x, t)+H_{0}^{U}(x, t)\right]\right] \\
=S^{-1}\left[u^{\alpha} S\left[a D_{x}^{3 \beta} \widetilde{U}_{0}(x)+6 a \widetilde{U}_{0}(x) D_{x}^{\gamma} \widetilde{U}_{0}(x)+2 b \widetilde{V}_{0}(x) D_{x}^{\delta} \widetilde{V}_{0}(x)\right]\right]
\end{array}
$$




$$
\begin{aligned}
& =\frac{t^{\alpha}}{\Gamma(\alpha+1)}\left(a D_{x}^{3 \beta} \widetilde{U}_{0}(x)+6 a \widetilde{U}_{0}(x) D_{x}^{\gamma} \widetilde{U}_{0}(x)+2 b \widetilde{V}_{0}(x) D_{x}^{\delta} \widetilde{V}_{0}(x)\right)=\frac{t^{\alpha}}{\Gamma(\alpha+1)} \widetilde{U}_{1}(x), \\
& V_{1}(x, t)=S^{-1}\left[v^{\alpha} S\left[-D_{x}^{3 \lambda} V_{0}(x, t)+H_{0}^{V}(x, t)\right]\right]=S^{-1}\left[v^{\alpha} S\left[-D_{x}^{3 \lambda} \widetilde{V}_{0}(x)-3 \widetilde{U}_{0} D_{x}^{\tau} \widetilde{V}_{0}(x)\right]\right] \\
& =\frac{t^{\alpha}}{\Gamma(\alpha+1)}\left(-D_{x}^{3 \lambda} \widetilde{V}_{0}(x)-3 \widetilde{U}_{0} D_{x}^{\tau} \widetilde{V}_{0}(x)\right)=\frac{t^{\alpha}}{\Gamma(\alpha+1)} \widetilde{V}_{1}(x) .
\end{aligned}
$$

Generally, we get

$$
\begin{aligned}
p^{k}: & U_{k}(x, t) \\
& =\frac{t^{k \alpha}}{\Gamma(k \alpha+1)}\left\{a D_{x}^{3 \beta} \widetilde{U}_{k-1}(x)+\sum_{i=0}^{k-1} \frac{\Gamma((k-1) \alpha+1)}{\Gamma(i \alpha+1) \Gamma((k-i-1) \alpha+1)}\left(6 a \widetilde{U}_{i}(x) D_{x}^{\gamma} \widetilde{U}_{k-1-i}(x)+2 b \widetilde{V}_{i}(x) D_{x}^{\delta} \widetilde{V}_{k-1-i}(x)\right)\right\} \\
& =\frac{t^{k \alpha}}{\Gamma(k \alpha+1)} \widetilde{U}_{k}(x) \\
& V_{k}(x, t)=\frac{t^{k \alpha}}{\Gamma(k \alpha+1)}\left(-D_{x}^{3 \lambda} \widetilde{V}_{k-1}(x)-\sum_{i=0}^{k-1} \frac{\Gamma((k-1) \alpha+1)}{\Gamma(i \alpha+1) \Gamma((k-i-1) \alpha+1)} 3 \widetilde{U}_{i}(x) D_{x}^{\tau} \widetilde{V}_{k-1-i}(x)\right)=\frac{t^{k \alpha}}{\Gamma(k \alpha+1)} \widetilde{V}_{k}(x),
\end{aligned}
$$

where

$$
\begin{aligned}
& \widetilde{U}_{k}(x)=a D_{x}^{3 \beta} \widetilde{U}_{k-1}(x) \\
& +\sum_{i=0}^{k-1} \frac{\Gamma((k-1) \alpha+1)}{\Gamma(i \alpha+1) \Gamma((k-i-1) \alpha+1)} 6 a \widetilde{U}_{i}(x) \\
& \cdot D_{x}^{\gamma} \widetilde{U}_{k-1-i}(x)+2 b \widetilde{V}_{i}(x) D_{x}^{\delta} \widetilde{V}_{k-1-i}(x), \\
& \widetilde{V}_{k}(x)=-D_{x}^{3 \lambda} \widetilde{V}_{k-1}(x) \\
& \quad-\sum_{i=0}^{k-1} \frac{\Gamma((k-1) \alpha+1)}{\Gamma(i \alpha+1) \Gamma((k-i-1) \alpha+1)} 3 \widetilde{U}_{i}(x) \\
& \cdot D_{x}^{\tau} \widetilde{V}_{k-1-i}(x) .
\end{aligned}
$$

Thus, the series solution of (65) is

$$
\begin{aligned}
& U(x, t)=\sum_{k=0}^{\infty} U_{k}(x, t)=\sum_{k=0}^{\infty} \widetilde{U}_{k}(x) \frac{t^{k \alpha}}{\Gamma(k \alpha+1)}, \\
& V(x, t)=\sum_{k=0}^{\infty} V_{k}(x, t)=\sum_{k=0}^{\infty} \widetilde{V}_{k}(x) \frac{t^{k \alpha}}{\Gamma(k \alpha+1)} .
\end{aligned}
$$

4.5. The Time-Space Fractional Coupled Whitham-BroerKaup (WBK) System. Under the Boussinesq approximation, Whitham [41], Broer [42], and Kaup [43] obtained the following nonlinear WBK system. In this subsection, we construct the approximate solution by the HPST method to the time-space fractional coupled WBK system.
Consider the time-space fractional coupled WBK system

$$
\begin{array}{r}
D_{t}^{\alpha} U+U D_{x}^{\beta} U+D_{x}^{\gamma} V+a D_{x}^{2 \delta} U=0, \\
D_{t}^{\alpha} V+D_{x}^{\lambda}(U V)-a D_{x}^{2 \tau} V+b D_{x}^{3 \theta} U=0
\end{array}
$$

with respect to the initial conditions

$$
\begin{aligned}
& U(x, 0)=a_{0}(x), \\
& V(x, 0)=b_{0}(x),
\end{aligned}
$$

where $0<\alpha, \beta, \gamma, \lambda \leq 1,1 / 2<\delta, \tau \leq 1,2 / 3<\theta \leq 1$, $(x, t) \in \mathbb{R} \times[0, \infty), a, b \in \mathbb{R}$ denote different dispersive power, $U=U(x, t)$ is the field of horizontal velocity, and $V=V(x, t)$ is the height deviating equilibrium position of liquid.

Applying the Sumudu transform on both sides of (77) with the initial conditions, we obtain

$$
\begin{aligned}
& S[U(x, t)]=a_{0}(x)+u^{\alpha}\left(S \left[-U(x, t) D_{x}^{\beta} U(x, t)\right.\right. \\
& \left.\left.\quad-D_{x}^{\gamma} V(x, t)-a D_{x}^{2 \delta} U(x, t)\right]\right), \\
& S[V(x, t)]=b_{0}(x)+v^{\alpha}\left(S \left[-D_{x}^{\lambda}(U(x, t) V(x, t))\right.\right. \\
& \left.\left.\quad+a D_{x}^{2 \tau} V(x, t)-b D_{x}^{3 \theta} U(x, t)\right]\right) .
\end{aligned}
$$

The inverse Sumudu transform of (79) implies that

$$
\begin{aligned}
& U(x, t)=a_{0}(x)+S^{-1}\left[u ^ { \alpha } \left(S \left[-U(x, t) D_{x}^{\beta} U(x, t)\right.\right.\right. \\
& \left.\left.\left.-D_{x}^{\gamma} V(x, t)-a D_{x}^{2 \delta} U(x, t)\right]\right)\right]
\end{aligned}
$$




$$
\begin{aligned}
& V(x, t)=b_{0}(x)+S^{-1}\left[v ^ { \alpha } \left(S \left[-D_{x}^{\lambda}(U(x, t) V(x, t))\right.\right.\right. \\
& \left.\left.\left.\quad+a D_{x}^{2 \tau} V(x, t)-b D_{x}^{3 \theta} U(x, t)\right]\right)\right] .
\end{aligned}
$$

Using homotopy perturbation method, it leads to

$$
\begin{aligned}
& \sum_{n=0}^{\infty} p^{n} U_{n}(x, t)=a_{0}(x)+p \\
& \times S^{-1}\left[u ^ { \alpha } S \left[-D_{x}^{\gamma}\left(\sum_{n=0}^{\infty} p^{n} V_{n}(x, t)\right)\right.\right. \\
& \left.\left.\quad-a D_{x}^{2 \delta}\left(\sum_{n=0}^{\infty} p^{n} U_{n}(x, t)\right)-\sum_{n=0}^{\infty} p^{n} H_{n}^{U}(x, t)\right]\right] \\
& \sum_{n=0}^{\infty} p^{n} V_{n}(x, t)=b_{0}(x)+p \\
& \quad \times S^{-1}\left[v ^ { \alpha } S \left[a D_{x}^{2 \tau}\left(\sum_{n=0}^{\infty} p^{n} V_{n}(x, t)\right)\right.\right. \\
& \left.\left.\quad-b D_{x}^{3 \theta}\left(\sum_{n=0}^{\infty} p^{n} U_{n}(x, t)\right)-\sum_{n=0}^{\infty} p^{n} H_{n}^{V}(x, t)\right]\right]
\end{aligned}
$$

where $H_{n}^{U}(x, t)$ and $H_{n}^{V}(x, t)$ are polynomials of the nonlinear term and are given by

$$
\begin{aligned}
& \sum_{n=0}^{\infty} p^{n} H_{n}^{U}(x, t)=U(x, t) D_{x}^{\beta} U(x, t), \\
& \sum_{n=0}^{\infty} p^{n} H_{n}^{V}(x, t)=D_{x}^{\lambda}(V(x, t) U(x, t)) .
\end{aligned}
$$

Setting

$$
\begin{aligned}
& U_{n}(x, t):=\widetilde{U}_{n}(x) \frac{t^{n \alpha}}{\Gamma(n \alpha+1)}, \\
& V_{n}(x, t):=\widetilde{V}_{n}(x) \frac{t^{n \alpha}}{\Gamma(n \alpha+1)},
\end{aligned}
$$

then

$$
\begin{aligned}
& H_{n}^{U}(x, t) \\
& \quad=\sum_{i=0}^{n} \widetilde{U}_{i}(x) D_{x}^{\beta} \widetilde{U}_{n-i}(x) \frac{t^{n \alpha}}{\Gamma(i \alpha+1) \Gamma((n-i) \alpha+1)}, \\
& H_{n}^{V}(x, t) \\
& \quad=D_{x}^{\lambda} \sum_{i=0}^{n} \widetilde{U}_{i}(x) \widetilde{V}_{n-i}(x) \frac{t^{n \alpha}}{\Gamma(i \alpha+1) \Gamma((n-i) \alpha+1)} .
\end{aligned}
$$

Comparing the coefficients of $p$, this gives

$$
\begin{aligned}
p^{0}: & U_{0}(x, t)=\widetilde{U}_{0}(x)=a_{0}(x), \\
& V_{0}(x, t)=\widetilde{V}_{0}(x)=b_{0}(x), \\
p^{1}: \quad & U_{1}(x, t)=S^{-1}\left[u^{\alpha} S\left[-D_{x}^{\gamma} V_{0}(x, t)-a D_{x}^{2 \delta} U_{0}(x, t)-H_{0}^{U}(x, t)\right]\right] \\
& =S^{-1}\left[u^{\alpha} S\left[-D_{x}^{\gamma} \widetilde{V}_{0}(x)-a D_{x}^{2 \delta} \widetilde{U}_{0}(x)-\widetilde{U}_{0}(x) D_{x}^{\beta} \widetilde{U}_{0}(x)\right]\right] \\
& =\frac{t^{\alpha}}{\Gamma(\alpha+1)}\left(-D_{x}^{\gamma} \widetilde{V}_{0}(x)-a D_{x}^{2 \delta} \widetilde{U}_{0}(x)-\widetilde{U}_{0}(x) D_{x}^{\beta} \widetilde{U}_{0}(x)\right)=\frac{t^{\alpha}}{\Gamma(\alpha+1)} \widetilde{U}_{1}(x), \\
& V_{1}(x, t)=S^{-1}\left[v^{\alpha} S\left[a D_{x}^{2 \tau} V_{0}(x, t)-b D_{x}^{3 \theta} U_{0}(x, t)-H_{0}^{V}(x, t)\right]\right] \\
& =S^{-1}\left[v^{\alpha} S\left[a D_{x}^{2 \tau} \widetilde{V}_{0}(x)-b D_{x}^{3 \theta} \widetilde{U}_{0}(x)-D_{x}^{\lambda}\left(\widetilde{U}_{0}(x) \widetilde{V}_{0}(x)\right)\right]\right] \\
& =\frac{t^{\alpha}}{\Gamma(\alpha+1)}\left(a D_{x}^{2 \tau} \widetilde{V}_{0}(x)-b D_{x}^{3 \theta} \widetilde{U}_{0}(x)-D_{x}^{\lambda}\left(\widetilde{U}_{0}(x) \widetilde{V}_{0}(x)\right)\right)=\frac{t^{\alpha}}{\Gamma(\alpha+1)} \widetilde{V}_{1}(x) .
\end{aligned}
$$

Generally, we have

$$
\begin{aligned}
p^{k}: & U_{k}(x, t)=\frac{t^{k \alpha}}{\Gamma(k \alpha+1)}\left\{-D_{x}^{\gamma} \widetilde{V}_{k-1}(x)-a D_{x}^{2 \delta} \widetilde{U}_{k-1}(x)-\sum_{i=0}^{k-1} \frac{\Gamma((k-1) \alpha+1)}{\Gamma(i \alpha+1) \Gamma((k-i-1) \alpha+1)} \widetilde{U}_{i}(x) D_{x}^{\beta} \widetilde{U}_{k-1-i}(x)\right\} \\
& =\frac{t^{k \alpha}}{\Gamma(k \alpha+1)} \widetilde{U}_{k}(x),
\end{aligned}
$$




$$
\begin{aligned}
& V_{k}(x, t)=\frac{t^{k \alpha}}{\Gamma(k \alpha+1)}\left\{a D_{x}^{2 \tau} \widetilde{V}_{k-1}(x)-b D_{x}^{3 \theta} \widetilde{U}_{k-1}(x)-\sum_{i=0}^{k-1} \frac{\Gamma((k-1) \alpha+1)}{\Gamma(i \alpha+1) \Gamma((k-i-1) \alpha+1)} D_{x}^{\lambda}\left(\widetilde{U}_{i}(x) \widetilde{V}_{k-1-i}(x)\right)\right\} \\
& =\frac{t^{k \alpha}}{\Gamma(k \alpha+1)} \widetilde{V}_{k}(x),
\end{aligned}
$$

where

$$
\begin{aligned}
& \widetilde{U}_{k}(x)=-D_{x}^{\gamma} \widetilde{V}_{k-1}(x)-a D_{x}^{2 \delta} \widetilde{U}_{k-1}(x) \\
& -\sum_{i=0}^{k-1} \frac{\Gamma((k-1) \alpha+1)}{\Gamma(i \alpha+1) \Gamma((k-i-1) \alpha+1)} \widetilde{U}_{i}(x) \\
& \cdot D_{x}^{\beta} \widetilde{U}_{k-1-i}(x), \\
& \widetilde{V}_{k}(x)=a D_{x}^{2 \tau} \widetilde{V}_{k-1}(x)-b D_{x}^{3 \theta} \widetilde{U}_{k-1}(x) \\
& \quad-\sum_{i=0}^{k-1} \frac{\Gamma((k-1) \alpha+1)}{\Gamma(i \alpha+1) \Gamma((k-i-1) \alpha+1)} D_{x}^{\lambda}\left(\widetilde{U}_{i}(x)\right. \\
& \left.\cdot \widetilde{V}_{k-1-i}(x)\right) .
\end{aligned}
$$

Hence, the series solution of (77) is

$$
\begin{aligned}
& U(x, t)=\sum_{k=0}^{\infty} U_{k}(x, t)=\sum_{k=0}^{\infty} \widetilde{U}_{k}(x) \frac{t^{k \alpha}}{\Gamma(k \alpha+1)}, \\
& V(x, t)=\sum_{k=0}^{\infty} V_{k}(x, t)=\sum_{k=0}^{\infty} \widetilde{V}_{k}(x) \frac{t^{k \alpha}}{\Gamma(k \alpha+1)}
\end{aligned}
$$

Remark 10. When alpha $=\tau=\delta=\lambda=\theta=1, \beta \neq 0$, and $\gamma=0,(77)$ reduces to the classical long-wave system that describes the shallow water wave with diffusion.

Remark 11. When $\alpha=\tau=\delta=\lambda=\theta=1, \beta=0$, and $\gamma=1$, (77) reduces to the variant Boussinesq system.

\section{Concluding Remarks}

In this paper, we apply the HPSTM to the nonlinear timespace fractional coupled equations. Applying the HPSTM, we can obtain analytic and approximate solutions to different coupled systems, for example, the coupled Burgers system, the coupled KdV system, the generalized Hirota-Satsuma coupled KdV system, the coupled WBK system, and the coupled shallow water system. The advantage of the HPSTM is its capability of combining two powerful methods for obtaining exact and approximate analytical solutions for nonlinear system. It provides the solutions in terms of convergent series with easily computable components in a direct way without using linearization, perturbation, or restrictive assumptions. The numerical results indicate that this method is effective and simple in constructing analytic or approximate solutions to fractional coupled systems.

\section{Conflicts of Interest}

The authors declare that there are no conflicts of interest regarding the publication of this paper.

\section{Acknowledgments}

The authors thank Dr. Yixian Gao for many useful suggestions and help. This research was supported in part by NSFC Grants 201114110, 11571065, 11671071, and 11401089, NSFJL Grants 20160520094JH and 20170101044JC, EDJLP Grant TTKH20170904KJ, and the Fundamental Research Funds for the Central Universities, 2412017FZ005.

\section{References}

[1] B. Ross, "The development of fractional calculus 1695-1900," Historia Mathematica, vol. 4, pp. 75-89, 1977.

[2] K. S. Miller and B. Ross, An Introduction to the Fractional Calculus and Fractional Differential Equations, A Wiley-Interscience Publication, John Wiley \& Sons, New York, NY, USA, 1993.

[3] N. Sebaa, Z. E. A. Fellah, W. Lauriks, and C. Depollier, "Application of fractional calculus to ultrasonic wave propagation in human cancellous bone," Signal Processing, vol. 86, no. 10, pp. 2668-2677, 2006.

[4] K. Assaleh and W. M. Ahmad, "Modeling of speech signals using fractional calculus," in Proceedings of the 2007 9th International Symposium on Signal Processing and Its Applications, ISSPA, UAE, February 2007.

[5] R. L. Magin and M. Ovadia, "Modeling the cardiac tissue electrode interface using fractional calculus," in Proceedings of the 2nd IFAC Workshop on Fractional Differentiation and Its Applications, FDA, pp. 302-307, July 2006.

[6] J. I. Suarez, B. M. Vinagre, A. J. Calderon, C. A. Monje, and Y. Q. Chen, Using Fractional Calculus for Lateral and Longitudinal Control of Autonomous Vehicles, Springer, Berlin, Germany, 2003.

[7] V. V. Kulish and J. L. Lage, "Application of fractional calculus to fluid mechanics," Journal of Fluids Engineering, vol. 124, no. 3, pp. 803-806, 2002.

[8] N. Bildik and A. Konuralp, "The use of variational iteration method, differential transform method and Adomian decomposition method for solving different types of nonlinear partial differential equations," International Journal of Nonlinear Sciences and Numerical Simulation, vol. 7, no. 1, pp. 65-70, 2006.

[9] K. K. Kataria and P. Vellaisamy, "Saigo space-time fractional poisson process via adomian decomposition method," Statistics \& Probability Letters, vol. 129, pp. 69-80, 2017.

[10] E. Yuluklu, "Comparison differential transform method with Adomian decomposition method for nonlinear initial value problems," Proceedings of the Jangjeon Mathematical Society. 
Memoirs of the Jangjeon Mathematical Society, vol. 20, no. 1, pp. 95-104, 2017.

[11] Z. M. Odibat and S. Momani, "Application of variational iteration method to nonlinear differential equations of fractional order," International Journal of Nonlinear Sciences and Numerical Simulation, vol. 7, no. 1, pp. 27-34, 2006.

[12] Z. Odibat and S. Momani, "A generalized differential transform method for linear partial differential equations of fractional order," Applied Mathematics Letters, vol. 21, no. 2, pp. 194-199, 2008.

[13] F. Xu, Y. Gao, X. Yang, and H. Zhang, "Construction of fractional power series solutions to fractional boussinesq equations using residual power series method," Mathematical Problems in Engineering, vol. 2016, Article ID 5492535, 15 pages, 2016.

[14] W. Yin, F. Xu, W. Zhang, and Y. Gao, "Asymptotic expansion of the solutions to time-space fractional kuramoto-sivashinsky equations," Advances in Mathematical Physics, vol. 2016, Article ID 4632163, 9 pages, 2016.

[15] F. Xu, Y. Gao, and W. Zhang, "Construction of analytic solution for time-fractional boussinesq equation using iterative method," Advances in Mathematical Physics, vol. 2015, Article ID 506140, 7 pages, 2015.

[16] Z. Odibat and S. Momani, "Modified homotopy perturbation method: application to quadratic Riccati differential equation of fractional order," Chaos, Solitons \& Fractals, vol. 36, no. 1, pp. 167-174, 2008.

[17] M. Dehghan, J. Manafian, and A. Saadatmandi, "Solving nonlinear fractional partial differential equations using the homotopy analysis method," Numerical Methods for Partial Differential Equations, vol. 26, no. 2, pp. 448-479, 2010.

[18] Y. Yildirim, E. Yasar, and A. . Adem, "A multiple exp-function method for the three model equations of shallow water waves," Nonlinear Dynamics, vol. 89, no. 3, pp. 2291-2297, 2017.

[19] E. M. Zayed, Y. A. Amer, and A.-G. Al-Nowehy, "The modified simple equation method and the multiple exp-function method for solving nonlinear fractional Sharma-Tasso-Olver equation," Acta Mathematicae Applicatae Sinica, vol. 32, no. 4, pp. 793-812, 2016.

[20] W.-X. Ma and J.-H. Lee, "A transformed rational function method and exact solutions to the $3+1$ dimensional Jimbo-Miwa equation," Chaos, Solitons \& Fractals, vol. 42, no. 3, pp. 13561363, 2009.

[21] M. Mirzazadeh, "A couple of solutions to a $(3+1)$-dimensional generalized KP equation with variable coefficients by extended transformed rational function method," Electronic Journal of Mathematical Analysis and Applications. EJMAA, vol. 3, no. 1, pp. 188-194, 2015.

[22] H. Zhang and W.-X. Ma, "Extended transformed rational function method and applications to complexiton solutions," Applied Mathematics and Computation, vol. 230, pp. 509-515, 2014.

[23] S. Choudhary and V. Daftardar-Gejji, "Invariant subspace method: a tool for solving fractional partial differential equations," Fractional Calculus and Applied Analysis, vol. 20, no. 2, pp. 477-493, 2017.

[24] W.-X. Ma, "A refined invariant subspace method and applications to evolution equations," Science China Mathematics, vol. 55, no. 9, pp. 1769-1778, 2012.

[25] J. Singh, D. Kumar, and Sushila, "Homotopy perturbation Sumudu transform method for nonlinear equations," Advances in Theoretical and Applied Mechanics, vol. 4, no. 4, pp. 165-175, 2011.

[26] K. Ait Touchent and F. B. Belgacem, "Nonlinear fractional partial differential equations systems solutions through a hybrid homotopy perturbation sumudu transform method," Nonlinear Studies. The International Journal, vol. 22, no. 4, pp. 591-600, 2015.

[27] D. Sharma, P. Singh, and S. Chauhan, "Homotopy perturbation transform method with he's polynomial for solution of coupled nonlinear partial differential equations," Nonlinear Engineering, vol. 5, no. 1, pp. 17-23, 2016.

[28] S. Tuluce Demiray, H. Bulut, and F. B. M. Belgacem, "Sumudu transform method for analytical solutions of fractional type ordinary differential equations," Mathematical Problems in Engineering, vol. 2015, Article ID 131690, 6 pages, 2015.

[29] G. K. Watugala, "Sumudu transform-a new integral transform to solve differential equations and control engineering problems," Journal of Mathematics in Industry, vol. 6, no. 4, pp. 319329, 1998.

[30] M. A. Asiru, "Further properties of the sumudu transform and its applications," International Journal of Mathematical Education in Science and Technology, vol. 33, no. 3, pp. 441-449, 2002.

[31] Q. D. Katatbeh and F. B. M. Belgacem, "Applications of the Sumudu transform to fractional differential equations," Nonlinear Studies, vol. 18, no. 1, pp. 99-112, 2011.

[32] J.-H. He, "Homotopy perturbation technique," Computer Methods Applied Mechanics and Engineering, vol. 178, no. 3-4, pp. 257-262, 1999.

[33] R. A. Kraenkel, J. G. Pereira, and M. A. Manna, "Nonlinear surface-wave excitations in the Bénard-Marangoni system," Physical Review A: Atomic, Molecular and Optical Physics, vol. 46, no. 8, pp. 4786-4790, 1992.

[34] V. Gurarie and A. Migdal, "Instantons in the Burgers equation," Physical Review E: Statistical, Nonlinear, and Soft Matter Physics, vol. 54, no. 5, pp. 4908-4914, 1996.

[35] Y. Wu, X. Geng, X. Hu, and S. Zhu, "A generalized HirotaSatsuma coupled Korteweg-de Vries equation and Miura transformations," Physics Letters A, vol. 255, no. 4-6, pp. 259-264, 1999.

[36] X. Shan and H. Chen, "Lattice Boltzmann model for simulating flows with multiple phases and components," Physical Review E: Statistical, Nonlinear, and Soft Matter Physics, vol. 47, no. 3, pp. 1815-1819, 1993.

[37] H. Chen, S. Kandasamy, S. Orszag, R. Shock, S. Succi, and V. Yakhot, "Extended Boltzmann kinetic equation for turbulent flows," Science, vol. 301, no. 5633, pp. 633-636, 2003.

[38] L. Giraud, D. D’Humières, and P. Lallemand, "A lattice Boltzmann model for Jeffreys viscoelastic fluid," EPL (Europhysics Letters), vol. 42, no. 6, pp. 625-630, 1998.

[39] R. Hirota and J. Satsuma, "Soliton solutions of a coupled Korteweg-de Vries equation," Physics Letters A, vol. 85, no. 8-9, pp. 407-408, 1981.

[40] E. Fan and H. Zhang, "New exact solutions to a solutions to a system of coupled KdV equations," Physics Letters A, vol. 245, no. 5, pp. 389-392, 1998.

[41] G. B. Whitham, "Variational methods and applications to water waves," in Hyperbolic Equations and Waves (Rencontres, Battelle Res. Inst., Seattle, Wash., 1968), pp. 153-172, Springer, Berlin, Germany, 1970. 
[42] L. J. F. Broer, "Approximate equations for long water waves," Applied Scientific Research, vol. 31, no. 5, pp. 377-395, 1975.

[43] D. J. Kaup, "A higher-order water-wave equation and the method for solving it," Progress of Theoretical and Experimental Physics, vol. 54, no. 2, pp. 396-408, 1975. 


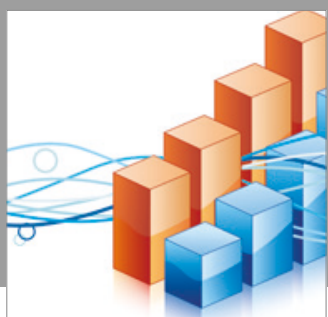

Advances in

Operations Research

vatersals

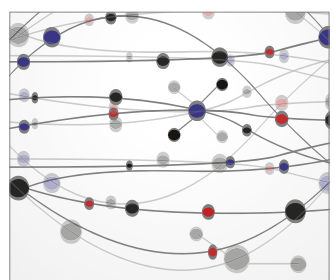

\section{The Scientific} World Journal
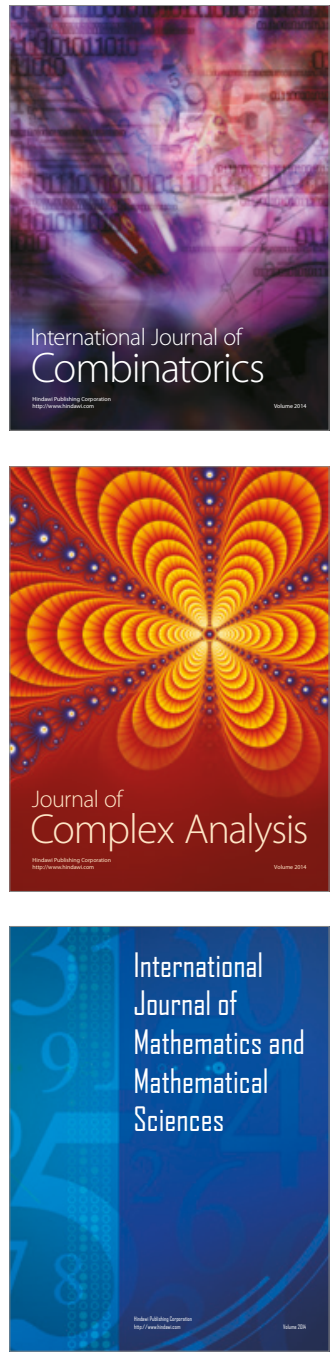
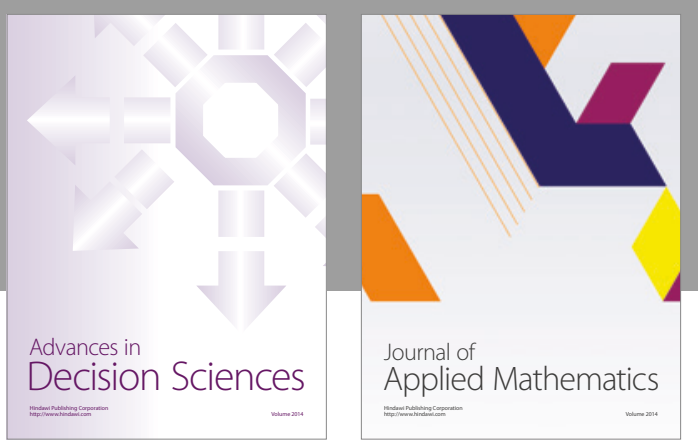

Algebra

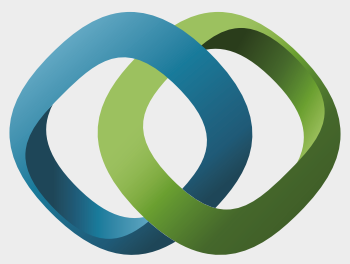

\section{Hindawi}

Submit your manuscripts at

https://www.hindawi.com
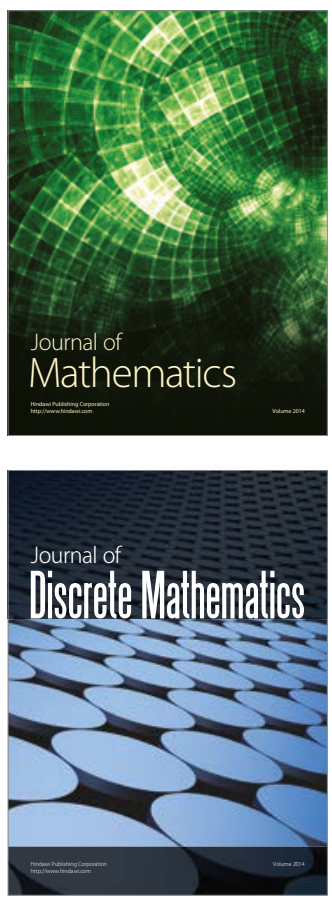

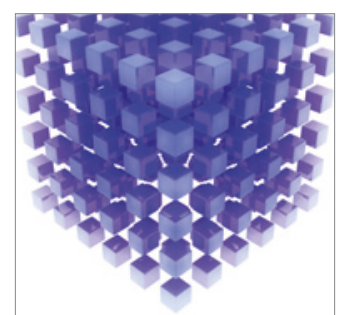

Mathematical Problems in Engineering
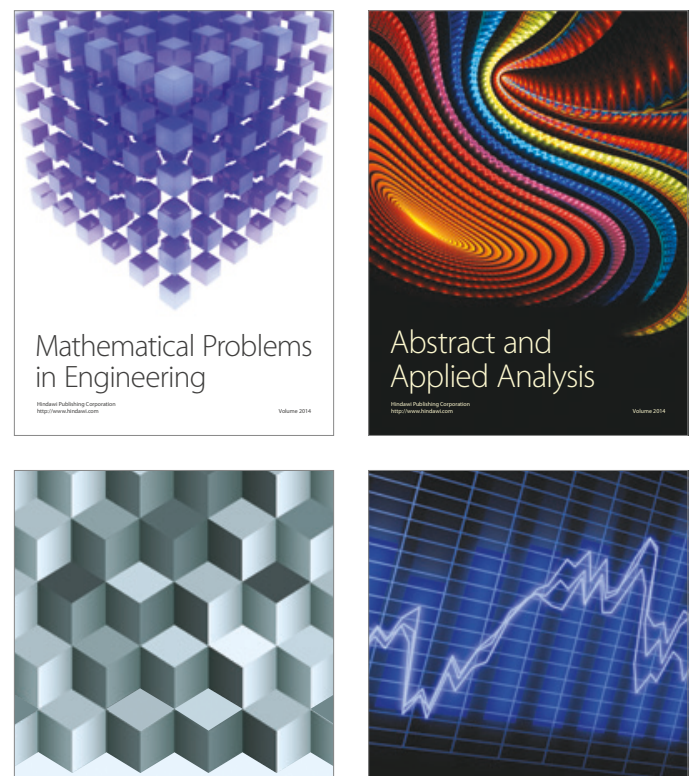

Journal of

Function Spaces

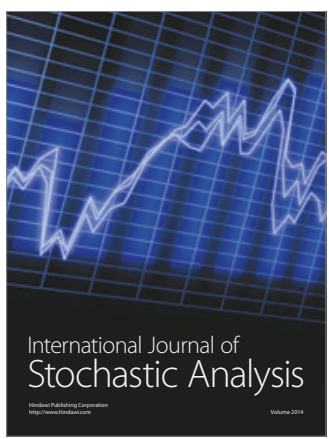

Probability and Statistics
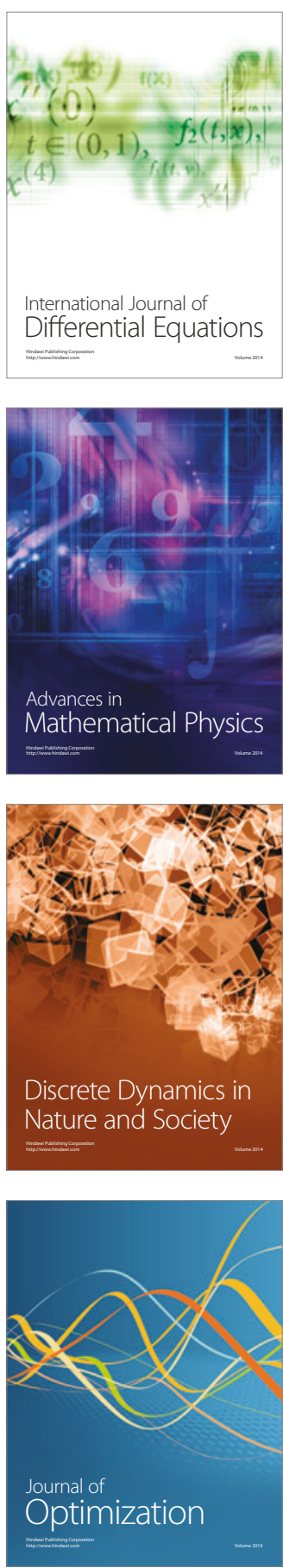